\title{
Qualité et essais de traitement des eaux en milieu périurbain : cas des eaux de forage des cantons Légbassito et Vakpossito (Togo)
}

\author{
Komlan FAMBI ${ }^{*}$, Massabalo AYAH ${ }^{1}$, Kossi Seyram SOSSOU ${ }^{2}$, Goumpoukini \\ BOGUIDO $^{1}$, Limam Moctar BAWA ${ }^{1}$ et Gbandi DJANEYE-BOUNDJOU ${ }^{1}$ \\ ${ }^{1}$ Laboratoire d'Hydrologie Appliquée et Environnement (LHAE-FDS/UL), 01 BP 1515, Lomé, Togo. \\ ${ }^{2}$ Laboratoire Eau Hydro-Systèmes et Agriculture (LEHSA), 2iE, 01 BP 594 Ouagadougou 01, Burkina Faso. \\ *Auteur correspondant; E-mail: komlanfambi@gmail.com
}

Received: 16-10-2019

Accepted: $18-12-2020$

Published: 28-02-2021

\section{RESUME}

L'objectif de ce travail est de contribuer à la potabilisation des eaux de forages dans les zones périurbaines de la ville de Lomé (Togo). L'évaluation de la qualité physicochimique et bactériologique a été réalisée sur 30 échantillons en utilisant les méthodes normalisées (AFNOR). Les essais d'élimination des nitrates par le charbon actif et de désinfection par le chlore pour la détermination du break-point ont été réalisés sur les eaux de plus forte teneur en nitrate dans chaque site étudié. Les résultats des enquêtes ont montré que les forages réalisés dans les zones périurbaines sont à $95 \%$ manuels. Les forages sont souvent implantés sans orientation technique à cause de la facilité qu'offre la formation sédimentaire. La profondeur varie de 25 à 35 $\mathrm{m}$. Les résultats ont montré que les forages sont réalisés dans un environnement insalubre, dépourvu d'infrastructure d'assainissement (plus de $80 \%$ ). Plus de $70 \%$ des échantillons d'eaux analysés ont des $\mathrm{pH}$ acide $(<6,5)$. Quatre-vingt-dix pour cent des échantillons d'eaux analysés ont des goûts salés, $87 \%$ des échantillons ne sont pas conformes à aux valeurs guides de l'OMS. La concentration des nitrates (élément toxique) et le fer (élément indésirable) sont très élevés, respectivement dans les proportions de $13 \%$ et $6 \%$ des eaux de forages analysées. La totalité des échantillons analysés est contaminée par des germes indicateurs d'origine fécale. Les échantillons d'eaux analysés ne sont pas conformes aux normes de potabilité des eaux de consommation. La contamination serait due prioritairement aux techniques de «foration » et à la maintenance périodique de la pompe. Les essais de traitement par le charbon actif et le chlore se sont révélés efficaces et constitueraient une des mesures à prendre en compte dans le traitement des eaux.

(C) 2021 International Formulae Group. All rights reserved.

Mots clés : Eau de forage, contamination, traitement, charbon actif, chlore.

\section{Quality and testing of water treatment in peri-urban areas: case of borehole water in the Légbassito and Vakpossito cantons (Togo)}

\begin{abstract}
The target of this study is to have a part in the preservation of sinking waters of Lomé city's suburbs areas. (Togo). The assessment of physicochemical quality and bacteriological has been carried out on 30 samples using standardized methods (AFNOR). Nitrate elimination tests with activated charcoal and
\end{abstract}


disinfecting chlorine for break-point determination have been carried out on waters of high nitrate content on each studied site. Surveys' results have shown that sinking, carried out in the suburbs' areas are of $95 \%$ manuals. Sinkings are often done without technical orientation due to the easy layer of sedimentary formation. The depth varies from 25 to $35 \mathrm{~m}$. Results showed that sinkings are carried out (over $80 \%$ ) in an unhealthy environment devoid of sanitation infrastructures. The $\mathrm{pH}$ is mostly acid $(<6,5)$. Waters are salted (about $90 \%)$ and $87 \%$ of samples are not standard to the WHO recommended values. Nitrates concentration (toxic element) and the iron (undesirable element) are very high, respectively in the proportions of $13 \%$ and $6 \%$ of tested sinking waters. The whole of tested samples are contaminated by germ indicators from faeces. Tested water samples are not standard to preservation norms of drinking water. Contamination might have resulted as a priority from «foration » techniques and periodic maintenance of the pump. Tests treatment with activated charcoal and the chlorine are effectively revealed and constitute one the measures to be taken into account in water-processing.

(C) 2021 International Formulae Group. All rights reserved.

Keywords: Water sinking, contamination, processing, activated charcoal, chlorine.

\section{INTRODUCTION}

L'eau destinée à la consommation humaine ne doit contenir ni substances chimiques dangereuses, ni germes nocifs pour la santé (OMS, 2011 ; 2iE, 2011 ; UE, 2015). La qualité de l'eau potable est liée à la lithologie et à l'assainissement autour des points d'eau. L'insuffisance des infrastructures d'approvisionnement en eau potable pour satisfaire les besoins d'hygiène de base (se laver les mains, faire sa toilette personnelle, etc.) entraine le recours à des sources d'eau plus facilement accessibles mais de qualité très peu connue (Coulibaly, 2005; EAATogo, 2016). Dans la ville de Lomé et ses banlieues (Togo), la plupart des ménages utilisent l'eau provenant des forages manuels et très peu réalisés dans les règles de l'art (EAA-Togo, 2016; Fambi, 2018). Les infrastructures en eau courante et l'accès à l'assainissement ne représentent qu'environ $32 \%$ et la demande d'extension du réseau de distribution d'eau potable est relativement chère en raison du coût de la vie (TdE et ONEA, 2015). La faible couverture en eau potable dans les zones périurbaines de Lomé et les comportements à risques des populations sont à l'origine des maladies telles que la fièvre typhoïde et paratyphoïde, la dysenterie amibienne et la poliomyélite (Coulibaly, 2005 ; OMS, 2011 ; Fambi, 2018). L'implantation, la réalisation et la surveillance d'un système d'alimentation en eau doivent être de rigueur afin de minimiser tout risque de pollution (Coulibaly, 2005 ; OMS, 2011 ;
ARC Ingénieur, 2016). Ce travail avait pour objectif d'évaluer la qualité physico-chimique et bactériologique des eaux de forages réalisés manuellement, d'identifier les sources de contamination des eaux, de procéder à quelques essais de traitement et de potabilisation de ces eaux par des agents physicochimiques et de dégager certaines causes de contamination des eaux.

\section{MATERIEL ET METHODES Zone d'étude}

La zone d'étude (cantons de Vakpossito et Légbassito) correspondant à la zone d'extension Nord-Ouest de la ville de Lomé, fait partie du Grand-Lomé (Préfecture du Golfe) (Groupement SGI, 2003; Ayah, 2016). Les deux cantons couvrent une superficie d'environ $43,51 \mathrm{~km}^{2}$ (Figure 1). La zone d'étude est caractérisée par un climat de type subéquatorial guinéen caractérisé par quatre saisons alternées : une grande saison sèche (de novembre à mi-mars), une grande saison pluvieuse (de mi-mars à mi-juillet), une petite saison sèche (d'août à septembre) et une petite saison pluvieuse couvrant le mois d'octobre. Elle est en général sous la dépendance de deux masses d'air : l'harmattan (sec et chaud) et la mousson (chaude et humide). La température moyenne est d'environ $27^{\circ} \mathrm{C}$ avec des écarts de 6 à 9 ${ }^{\circ} \mathrm{C}$ entre les minima et les maximas mensuels. En août, la température tombe jusqu'à $18^{\circ} \mathrm{C}$.

Les précipitations sont comprises en moyenne entre 80 et $950 \mathrm{~mm} / \mathrm{an}$. Néanmoins 
l'influence maritime adoucie la température et offre une humidité relativement élevée (> $75 \%)$ qui compense le déficit pluviométrique (Akouvi et al., 2008; Ayah, 2016). Le relief peu accidenté est caractérisé par des plateaux qui mettent en évidence la faible pente, du cordon littoral, du plateau de Lomé et des deux terrasses à galets du versant nord de la vallée du Zio. Le lit majeur du fleuve Zio correspond à la limite Nord-Est de la zone d'extension de la ville de Lomé et s'étale dans une vaste plaine inondable située entre 4 et 6 m d'altitude (Gnazou, 2008 ; Ayah, 2016).

La zone d'étude fait partie du bassin sédimentaire côtier du Togo caractérisée principalement par une série sédimentaire d'âge Méso-Cénozoïque (da Costa, 2005 ; Gnazou, 2008 ; Ségniagbéto, 2009). La nappe du Continental Terminal est particulièrement le plus exploitée. Selon les chiffres du dernier recensement en 2010, la zone d'étude compte plus de 56363 habitants. L'économie est essentiellement basée sur les activités de quelques branches de production de biens et de services (DGSCN, 2011 ; Ayah, 2016).

\section{Echantillonnage}

Les échantillons d'eau traités au cours de cette étude sont le résultat de la campagne d'échantillonnage de juillet 2017, après la prospection sur le terrain en juin 2017, qui a concerné le choix des forages, l'environnement et l'état de salubrité autour des points d'eau dans les cantons de Légbassito et de Vakpossito à travers l'observation sur le terrain et "l'interview direct » des propriétaires des points d'eau dans la zone d'étude (Figure 2). La distance entre les points d'eau, les sources potentielles de pollution (sanitaires, dépotoirs, eau usées, dépressions), les types de ressources en eau des ménages, la fréquence d'entretien des forages et les types de désinfectants utilisés et leurs doses ont également été des critères pris en compte sur le terrain. Ainsi, trente (30) forages privés à titre commercial ont été choisis pour cette étude dont quinze (15) échantillons d'eau dans chaque canton (canton de Légbassito et de Vakpossito).

Les niveaux statiques de l'eau dans les forages ont été également déterminés. Les principes et méthodes d'échantillonnage, d'analyses physicochimiques et bactériologiques décrits par l'Association Française de Normalisation (AFNOR) sur la «qualité et l'analyse de l'eau » ont été utilisés (AFNOR, 1997; Rejesk, 2002 ; Rodier, 2009). Ainsi, les échantillons d'eau destinés à l'analyse physicochimiques ont été prélevés dans des bouteilles plastiques en polyéthylène, propres, de capacité de 1,5 L. Pour les analyses bactériologiques, le matériel de prélèvement était constitué des flacons stériles $\left(121{ }^{\circ} \mathrm{C} / 25 \mathrm{mn}\right)$ de $0,5 \mathrm{~L}$ et d'un bruleur pour stériliser les robinets avant les prélèvements.

Tous les échantillons d'eau prélevés ont été acheminés aux laboratoires dans des glacières munies d'éléments réfrigérants à 4 ${ }^{\circ} \mathrm{C}$. Les analyses physicochimiques ont été réalisées au Laboratoire de Chimie des Eaux de la Faculté des Sciences de l'Université de Lomé et les analyses bactériologiques dans le Laboratoire de Microbiologie et de Contrôle de qualité des Denrées Alimentaires (LAMICODA) de l'École Supérieure des Techniques Biologiques et Alimentaires de l'Université de Lomé.

\section{Analyses physicochimiques}

Les paramètres physicochimiques notamment, la température $\left(\mathrm{T}^{\circ} \mathrm{C}\right)$, le $\mathrm{pH}$, la turbidité, la conductivité électrique (Cond.él.), le taux de sels dissouts (TDS), les anions majeurs $\left(\mathrm{HCO}_{3}^{-}, \mathrm{Cl}^{-}, \mathrm{SO}_{4}{ }^{2-},\right)$, les cations majeurs $\left(\mathrm{Ca}^{2+}, \mathrm{Mg}^{2+}, \mathrm{Na}^{+}, \mathrm{K}^{+}\right)$, la silice $\left(\mathrm{SiO}_{2}\right)$, les composés azotés $\left(\mathrm{NO}_{3}{ }^{-}\right)$et de phosphore $\left(\mathrm{PO}_{4}{ }^{2-}\right)$, le fer $\left(\mathrm{Fe}^{2+}\right)$, le manganèse $\left(\mathrm{Mn}^{2-}\right)$ et l'indice permanganate (IP) ont été déterminés (Tableau 1).

\section{Analyses bactériologiques}

Pour les germes contaminants des eaux, la méthode par incorporation dans la gélose a été utilisée pour la recherche et le dénombrement de la flore mésophile totale (ou germes totaux), les coliformes totaux, les coliformes thermotolérants et les germes anaérobies sulfitoréducteurs (Rejesk, 2002; Ajibadé et al., 2008). Le Tableau 2 indique les germes recherchés, les méthodes d'analyse et de préparation des milieux de culture. 


\section{Test d'élimination des nitrates et demande en chlore de l'eau}

- L'élimination des nitrates a été réalisée pour les échantillons d'eau de forage dont les concentrations en nitrates sont les plus élevées en utilisant le charbon actif en poudre (CAP) comme adsorbant. Les caractéristiques du CAP utilisés sont présentées dans le Tableau 3 (Chen et al., 2011 ; Bhatnagar et Sillanpää, 2011 ; Tchakala, 2013; Balogoun, 2016). L'élimination des nitrates a été réalisée sur deux séries de six flacons de $100 \mathrm{ml}$ des échantillons de fortes concentrations en nitrate (concentration maximale à Légbassito et à Vakpossito) mis en contact avec des masses croissantes de CAP (de $0,5 \mathrm{~g}$ à $10 \mathrm{~g}$ ). Après un temps sous agitation magnétiques $(1 \mathrm{~h}, 2 \mathrm{~h}$, $3 \mathrm{~h}, 4 \mathrm{~h}, 6 \mathrm{~h}, 12 \mathrm{~h}, 18 \mathrm{~h}$ et $24 \mathrm{~h}$ ), la séparation solide/liquide des échantillons a été réalisée par filtration sous vide à l'aide d'une membrane de $0,45 \mu \mathrm{m}$ de porosité. Les quantités résiduelles en nitrates ont été ensuite déterminées pour chaque échantillon filtré.

- Pour déterminer la demande en chlore des échantillons d'eau sur lesquels les tests d'élimination des nitrates ont été réalisés, les temps de contact nécessaires entre l'eau et le chlore ont été préalablement mesurés ; puis la demande en chlore a été déterminée par la méthode au break point (NFT 90-038) (AFNOR, 1997; Rejesk, 2002; Tchakala, 2008). La consommation de chlore a été réalisée en appliquant à $\mathrm{pH}$ libre de l'eau des doses croissantes $0,5 \mathrm{mgCl}_{2} / \mathrm{L}$ à $3,0 \mathrm{mgCl}_{2} / \mathrm{L}$ pour un temps de contact de $2 \mathrm{~h}$.

\section{Analyses statistiques}

Les méthodes statistiques descriptives (calcul de moyennes, écart-types et le test de Kruskwal wallis $(\mathrm{p}<0,05)$ ) et celles en analyses multidimensionnelles des données (analyse en composantes principales) ont été utilisées pour l'interprétation des résultats analytiques. Le diagramme de Piper pour une visualisation globale des faciès hydrochimiques (Simler, 2005) et les programmes informatiques comme Excel et le logiciel Xlstat (Fahmy, 2006) pour la détermination des relations entre les différents paramètres ont été utilisés ont été également utilisés (Simler, 2005 ; Fahmy, 2006).

Tableau 1 : Paramètres physicochimiques, matériel et méthodes d'analyses.

\begin{tabular}{|c|c|c|c|c|}
\hline Paramètres & Méthodes & Norme/référence & $\begin{array}{c}\text { Précision/ } \\
\text { Limite de détection }\end{array}$ & Matériel \\
\hline Localisation & Positionnement & - & $\pm 10 \mathrm{~m}$ & GPS Type "Garmin" \\
\hline Profondeur & $\begin{array}{l}\text { Sonde à câble lesté } \\
\text { de } 300 \mathrm{~m}\end{array}$ & - & $\pm 10,1 \mathrm{~mm}$ & $\begin{array}{l}\text { Solinst, Tag Line } \\
\text { Modèle } 103\end{array}$ \\
\hline $\begin{array}{l}\text { Température } \\
\mathrm{pH} \\
\text { Cond.él. }\end{array}$ & Electrométrie & $\begin{array}{l}\text { NFT } 90-100 \\
\text { NF T 90-008 } \\
\text { NF T } 90-031\end{array}$ & $\begin{array}{c} \pm 0,01{ }^{\circ} \mathrm{C} \\
\pm 0,01 \mathrm{upH} \\
0,5 \%\end{array}$ & $\begin{array}{c}\text { Multimètre } \\
\text { "Type WTW Multi 3420" }\end{array}$ \\
\hline Turbidité & Turbi dimètre & NF T 90-033 & $\pm 0.01 \mathrm{NTU}$ & $\begin{array}{l}\text { Turbidimètre } \\
\text { "Type DRT100B model } \\
\text { 20012" }\end{array}$ \\
\hline $\mathrm{HCO}_{3}^{-}$ & Acidimétrie & NF T 90-036 & $\pm 0,25 \mathrm{mg} / \mathrm{L}$ & \\
\hline $\begin{array}{l}\mathrm{Ca}^{2+} \\
\mathrm{Mg}^{2+}\end{array}$ & Complexométrie & NF T 90-016 & $\begin{array}{c} \pm 0,5 \mathrm{mg} / \mathrm{L} \\
\pm 0,24 \mathrm{mg} / \mathrm{L}\end{array}$ & Verrerie de laboratoire \\
\hline $\begin{array}{l}\mathrm{Cl}^{-} \\
\mathrm{IP}\end{array}$ & $\begin{array}{l}\text { Argentimétrie } \\
\text { Basique/Chaud }\end{array}$ & $\begin{array}{l}\text { NF T } 90-014 \\
\text { EN ISO } 8467\end{array}$ & $\begin{array}{l} \pm 0,5 \mathrm{mg} / \mathrm{L} \\
\pm 0,5 \mathrm{mg} / \mathrm{L}\end{array}$ & \\
\hline $\begin{array}{l}\mathrm{Na}^{+} \\
\mathrm{K}^{+}\end{array}$ & Absorption atomique & NF T 90-20 & $\begin{array}{l} \pm 0.04 \mathrm{mg} / \mathrm{L} \\
\pm 0.02 \mathrm{mg} / \mathrm{L}\end{array}$ & $\begin{array}{l}\text { Photomètre à flamme } \\
\text { "Type JENWAY PFP7" }\end{array}$ \\
\hline $\mathrm{SiO}_{2}$ & & NF T 90007 & & \\
\hline $\mathrm{NO}_{3}^{-}$ & Spectrométrie & NF T 90-012 & 1 à $2 \%$ & $\begin{array}{l}\text { Spectromètre UV/Visible } \\
\text { "Type JANWAY 6705" }\end{array}$ \\
\hline $\begin{array}{l}\mathrm{SO}_{4}^{2-} \\
\mathrm{Fe}^{2+}\end{array}$ & Néphélométrie & $\begin{array}{l}\text { NF T 90-009 } \\
\text { NF T 90-017 }\end{array}$ & & \\
\hline $\mathrm{Mn}^{2+}$ & Spectrométrie & NF T 90-024 & 1 à $2 \%$ & $\begin{array}{l}\text { Spectromètre UV/Visible } \\
\text { "Type JANWAY 6705" }\end{array}$ \\
\hline Chlore $\left(\mathrm{Cl}_{2}\right)$ & & NF T 90-037 & & \\
\hline
\end{tabular}




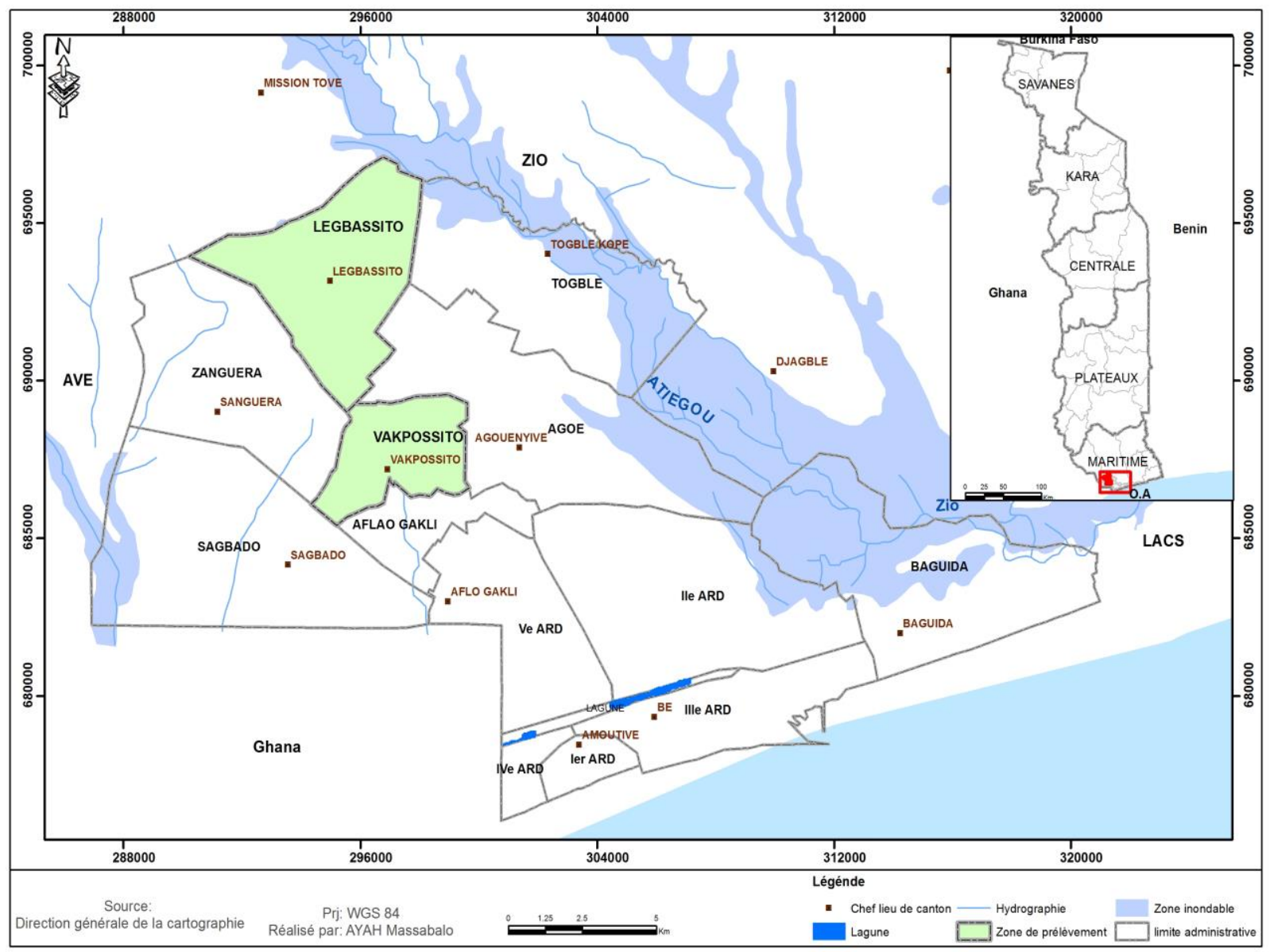

Figure 1 : Localisation de la zone d'étude.

Tableau 2 : Germes recherchés et méthodes de dénombrement dans les eaux analysées.

\section{Germes recherchés Milieu de culture}

Germes totaux

Coliformes totaux

C. thermotolérants

E. coli

ASR

VRBL

Brillance E. coli
Méthodes

NF V08-051, Fév. 1999

NF V08-050, Déc. 1992

NF V08-016, Déc. 1992

(CM 1046) Brilliance (*)

XP V08-061, Av. 1996

\section{Condition d'incubation}

$30{ }^{\circ} \mathrm{C} / 72 \mathrm{~h}$

$30{ }^{\circ} \mathrm{C} / 24 \mathrm{~h}$

$44{ }^{\circ} \mathrm{C} / 24 \mathrm{~h}$

$37{ }^{\circ} \mathrm{C} / 72 \mathrm{~h}$

$44{ }^{\circ} \mathrm{C} / 48 \mathrm{~h}$

ASR : Germes anaérobies sulfitoréducteurs ; PCA : Plate Count Agar ; VRBL : Violet Red Bile Lactose Agar ;

TSN : Tryptone Sulfite Néomycine ; $\left(^{*}\right)$ code CM 1046 (Brilliance ${ }^{\mathrm{TM}}$ E. coli/coliform selective agar). 


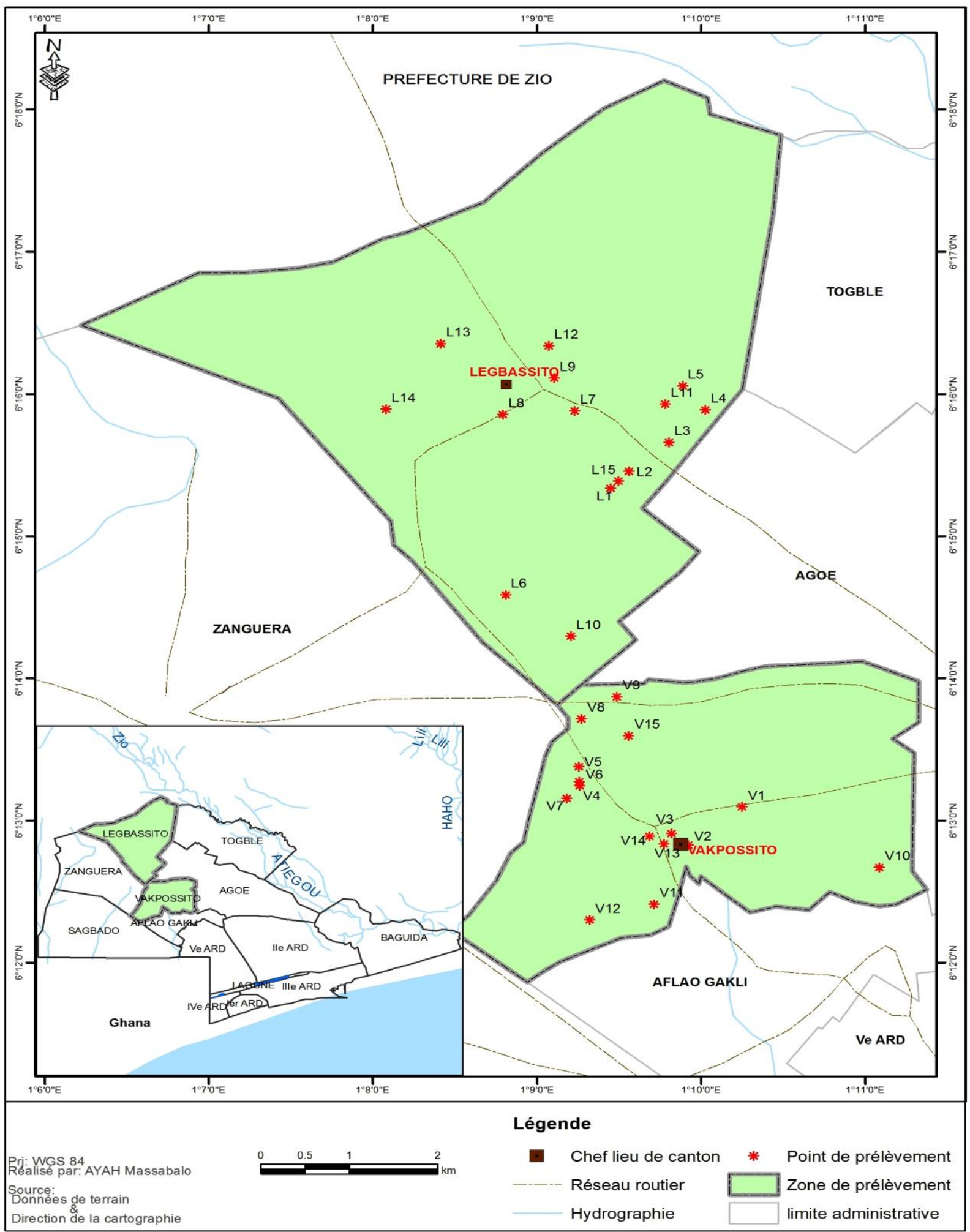

Figure 2: Localisation des sites de prélèvement. 
Tableau 3 : Quelques caractéristiques du charbon actif utilisé.

\begin{tabular}{lc}
\hline Paramètres & Caractéristiques \\
\hline Type & Charbon actif poudre $(\mathrm{CAP})$ \\
Matière première & Tourteau de Karité \\
Granulométrie $(\mu \mathrm{m})$ & 63 \\
Surface spécifique $\left(\mathrm{m}^{2} / \mathrm{g}\right)$ & 1450 \\
Volume poreux total $\left(\mathrm{cm}^{3} / \mathrm{g}\right)$ & 0,68 \\
\hline
\end{tabular}

\section{RESULTATS}

\section{Résultats de l'enquête}

L'enquête de juin 2017 sur l'état des points d'eau de forages privés dans les cantons Légbassito et Vakpossito a montré que la réalisation des forages privés pour la plupart est manuelle (Figure 3a). Le diagnostic sur le plan environnemental au niveau des trente (30) ménages (15 par canton) dont les eaux des forages ont fait l'objet de cette étude a montré en ce qui concerne l'utilisation de l'eau de forage, les traitements effectués et les fréquences d'entretien des forages, que la plupart des maisons sont en zone péri-urbaine avec des champs cultivés par endroit et où les infrastructures d'assainissement et de voiries sont pratiquement inexistantes : présence des dépotoirs incontrôlés, des eaux stagnantes dans un rayon de 50 à $100 \mathrm{~m}$ (Figures $3 \mathrm{~b}$ et $3 c$ ). La distance forage-latrines/puisards est en moyenne comprise entre $2 \mathrm{~m}$ à Vakpossito et $15 \mathrm{~m}$ à Légbassito. Par ailleurs, 57\% des forages sont proches des latrines/puisards (en moyenne $5 \mathrm{~m}$ ) et $43 \%$ sont conformes aux recommandations de l'OMS (distance minimale $15 \mathrm{~m}$ ) (OMS, 2011; 2iE, 2011 ; Fambi, 2018). Ainsi, 70\% des ménages utilisent les eaux de forages pour tout besoin (boisson, cuisson, hygiène corporelle, vaisselle, lessive, etc.), et $30 \%$ utilisent seulement comme eau de boisson, l'eau minérale en sachet communément appelée “pure-water" et l'eau de forage pour les autres besoins car elle serait impropre à la consommation. Le seul traitement réalisé par les ménages est le nettoyage du réservoir «polytank » à l'eau de Javel. En effet, 42\% des ménages enquêtées le font à des périodes non définit et à des doses aléatoires et $13 \%$ des ménages font le traitement à l'eau de Javel directement dans le forage au moment de l'entretien périodique (allant de 3 à 6 mois) de la pompe (Figure 3d). La profondeur des forages étudiés varie entre $24 \mathrm{~m}$ (Légbassito) et $35 \mathrm{~m}$ (Vakpossito), dont 7 sont des puits forés.

\section{Variation spatiale des paramètres physicochimiques des eaux}

Au total, trente (30) échantillons d'eau de forage ont été prélevés au cours de la campagne de juillet 2017 dans les cantons de Légbassito et de Vakpossito. Le Tableau 4 présente les résultats des paramètres physicochimiques des eaux dans les deux (02) sites étudiés (cantons de Légbassito et de Vakpossito). Les directives de l'OMS et de l'Union Européenne (OMS, 2011 ; 2iE, 2011 ; Chen et al., 2011) nous ont servies de référence pour l'interprétation des résultats.

Les résultats obtenus ont montré que les eaux ont des valeurs de $\mathrm{pH}$ comprises entre 4,9 (Vakpossito) et 6,6 (Légbassito). Les valeurs de la conductivité électrique varient entre $305 \mu \mathrm{S} / \mathrm{cm}$ (Vakpossito) et $1714 \mu \mathrm{S} / \mathrm{cm}$ (Légbassito) indiquant une salinité moyenne $(218 \mathrm{mg} / \mathrm{L})$ à forte $(1299 \mathrm{mg} / \mathrm{L})$. Les eaux 
étudiées sont en majorités claires avec des valeurs de la turbidité inférieure à 5 NTU, valeur recommandée par l'OMS. Elles contiennent par ailleurs de faibles concentrations en matières oxydables (inférieures à $2 \mathrm{mgO}_{2} / \mathrm{L}$ ). Cependant, on note des valeurs extrêmes dans les deux sites (3 NTU à Vakpossito et 8,8 NTU à Légbassito) (Figure 4d). Les valeurs limitent de la silice dans les deux sites sont comprises entre 16,5 et $170,36 \mathrm{mg} / \mathrm{L}$. Les éléments métalliques comme $\mathrm{Fe}$ et $\mathrm{Mn}$ présentent des concentrations pour la plupart faibles $(\leq 0,05$ $\mathrm{mg} / \mathrm{L}$ ). Ainsi, plus de $95 \%$ des échantillons ont des teneurs en fer total et en manganèse inférieures aux valeurs recommandées par l'OMS $(0,3 \mathrm{mgFe} / \mathrm{L}$ et $0,4 \mathrm{mgMn} / \mathrm{L})$, sauf à Légbassito où on enregistre des teneurs en fer total de $0,35 \mathrm{mg} / \mathrm{L}$ et $4,0 \mathrm{mg} / \mathrm{L}$. La faible valeur de l'alcalinité est enregistrée à Vakpossito $(12,2 \mathrm{mg} / \mathrm{L})$ où on a également observé une valeur maximale de 73,20 mg/L. Les concentrations en nitrates des eaux sont pour la plupart faibles. Cependant, le site de Légbassito enregistre la plus grande teneur en nitrates caractérisée par une valeur maximale de $154,1 \mathrm{mg} / \mathrm{L}$ (Figure 5b). Le site de Vakpossito présente une valeur atypique de $56,2 \mathrm{mg} / \mathrm{L}$ et une valeur extrême de 121,6 $\mathrm{mg} / \mathrm{L}$. Les teneurs en chlorures $\left(\mathrm{Cl}^{-}\right)$dans les sites sont en général élevées à Vakpossito avec une médiane de $330 \mathrm{mg} / \mathrm{L}$ tandis que dans la zone de Légbassito, les teneurs en chlorures sont relativement faibles par rapport à la valeur guide de l'OMS (250 mg/L) avec une médiane de $128 \mathrm{mg} / \mathrm{L}$ (Figure 5c). Pour les teneurs en sulfates, la plus grande valeur obtenue dans le cadre de cette étude est de $26,6 \mathrm{mg} / \mathrm{L}$ à Vakpossito. Tous les points d'eau présentent des teneurs inférieures à la valeur recommandée par l'OMS, soit $400 \mathrm{mg} / \mathrm{L}$. Les teneurs en $\mathrm{Na}$ dans les deux sites varient entre $21 \mathrm{mg} / \mathrm{L}$ à Légbassito et $220 \mathrm{mg} / \mathrm{L}$ à Vakpossito avec une forte variation au sein d'un même site (Figure 6a). Les médianes autour de $65 \mathrm{mg} / \mathrm{L}$ à Légbassito et $140 \mathrm{mg} / \mathrm{L}$ à Vakpossito avec des écarts significatifs des valeurs de la teneur en sodium respectivement de 21 à $142 \mathrm{mg} / \mathrm{L}$ et de 47 à $220 \mathrm{mg} / \mathrm{L}$. Les teneurs en potassium dans les différents sites sont faibles dans tous les sites avec une valeur atypique à Vakpossito (Figure 6b). Les teneurs en calcium $\left(\mathrm{Ca}^{2+}\right)$ dans les eaux varient entre $11,2 \mathrm{mg} / \mathrm{L}$ et $68 \mathrm{mg} / \mathrm{L}$ à Légbassito et $16 \mathrm{mg} / \mathrm{L}$ et $68 \mathrm{mg} / \mathrm{L}$ à Vakpossito avec des médianes respectives de $32 \mathrm{mg} / \mathrm{L}$ et $42 \mathrm{mg} / \mathrm{L}$. Les deux sites présentent un grand écart de variation donc une distribution peu variée (Figure 6c). Les teneurs en magnésium $\left(\mathrm{Mg}^{2+}\right)$ suivent les mêmes ordres d'étendue avec des médianes de $26 \mathrm{mg} / \mathrm{L}$ à Vakpossito et de $13 \mathrm{mg} / \mathrm{L}$ à Légbassito (Figure 6d).

\section{Variation spatiale des paramètres bactériologiques des eaux}

Les résultats des analyses bactériologiques des échantillons d'eau de forages sont présentés dans le Tableau 5. Les résultats ont montré que les eaux analysées dans les deux sites sont contaminées à des degrés divers par les germes totaux (68 à 4900 ufc/ml), les coliformes totaux (0 à 709 ufc/ml), les coliformes thermotolérants $44{ }^{\circ} \mathrm{C}$ ( 0 à $132 \mathrm{ufc} / \mathrm{ml}$ ), et les anaérobies sulfitoréducteurs ( 0 à $8 \mathrm{ufc} / \mathrm{ml}$ ). La qualité hygiénique des échantillons par rapport aux critères AFNOR a montré que 94,33\% des échantillons sont de qualité hygiénique non satisfaisante par rapport aux coliformes totaux dans le site de Vakpossito et 86,67\% dans le site de Légbassito.

\section{Analyses statistiques et représentions des eaux en familles hydrochimiques}

Les paramètres pris en compte pour l'Analyse en Composantes Principales (ACP) ont concerné la température $\left(\mathrm{T}^{\circ} \mathrm{C}\right)$, le $\mathrm{pH}$, la conductivité électrique (Cond.), le calcium $(\mathrm{Ca})$, le magnésium $(\mathrm{Mg})$, le sodium $(\mathrm{Na})$, le 
potassium $(\mathrm{K})$, le bicarbonate $\left(\mathrm{HCO}_{3}\right)$, le chlorure $(\mathrm{Cl})$, le sulfate $\left(\mathrm{SO}_{4}\right)$, le nitrate $\left(\mathrm{NO}_{3}\right)$, les germes totaux (GTo), les coliformes totaux (CTo), les distances foragelatrine (DFL), la profondeur (PrF) et l'oxydabilité au permanganate de potassium $\left(\mathrm{KMnO}_{4}\right)$. A partir de la matrice de corrélation présentée dans le Tableau 6 , on peut distinguer deux groupes d'éléments corrélés : les éléments très significativement corrélés avec un coefficient de corrélation supérieur ou égal à 0,55 , et les éléments significativement corrélés avec un coefficient de corrélation situé entre 0,45 et 0,55 . Ainsi, les représentations des données en cercles de corrélation (Tableau 6; Figure 7) montrent entre les axes F1-F2, une variabilité de $52,73 \%$ et $43,97 \%$ pour les axes F1-F3.

Le diagramme de Piper nous a permis de représenter sur un même graphique de nombreuses analyses avec des regroupements par famille et de faciès similaires (Simler, 2005). Les familles des eaux analysées sont présentées sur la Figure 8.

Ainsi, le digramme ternaire des anions montre que la majorité des points d'eau analysés est orientée vers le pôle chloruré, tandis que dans le diagramme ternaire des cations, la majorité des points est orienté vers le pôle sodique.

A partir de ces observations, deux familles ont été identifiées : une famille Chlorurée-sodique, c'est le cas des échantillons de Vakpossito où la majorité des eaux contient en proportion ionique plus de $95 \%$ de $\mathrm{Cl}^{-}$et près de $70 \%$ de $\mathrm{Na}^{+}$, et une famille bicarbonatée calcique pour les échantillons de Légbassito où la majorité des eaux contient plus de $50 \%$ de $\mathrm{Ca}^{2+}$ et 55 à $60 \%$ de $\mathrm{HCO}_{3}{ }^{-}$. Le diagramme de Piper a permis d'avoir une classification simple des eaux en fonction des ions majeurs contenus dans les eaux.

\section{Résultats des essais d'élimination des nitrates par le charbon actif}

Les résultats des essais d'élimination des nitrates par le charbon actif pour les deux échantillons fortement chargés en nitrate dans les cantons Légbassito $(154,1 \mathrm{mg} / \mathrm{L})$ et Vakpossito (121,62 $\mathrm{mg} / \mathrm{L})$ ont montré respectivement un abattement de $74,2 \%$ et de $80,6 \%$ pour une masse de charbon actif de 10 $\mathrm{g}$ après $24 \mathrm{~h}$ d'agitation (Figure 9 et Tableau 7).

\section{Résultats des essais de la demande en chlore}

Pour un traitement complet, des essais de désinfection ont été réalisés avec une eau de javel à $38,04 \mathrm{mgCl}_{2} / \mathrm{L}$ (soit $12{ }^{\circ} \mathrm{Chl}$ ). La demande en chlore des eaux a été réalisée sur les deux échantillons chargés en nitrate de Légbassito et de Vakpossito. Le temps de contact observé est de $2 \mathrm{~h}$. La charge bactérienne des deux échantillons chargés en nitrate (Légbassito et Vakpossito) a été déterminée avant et après le traitement au chlore.

Pour la mesure de la demande en chlore, la méthode par iodométrie a permis de déterminer le break point des deux échantillons ayant des teneurs maximales en nitrate à Légbassito et Vakpossito. Les courbes de break point obtenues ont montré que les doses de chlores appliquées au début sont détruites (Figure 10). Ainsi, la demande en chlore des deux échantillons d'eau de forage des cantons Légbassito et Vakpossito étaient respectivement de $1,35 \mathrm{mgCl}_{2} / \mathrm{L}$ et de $1,30 \mathrm{mgCl}_{2} / \mathrm{L}$. Ces valeurs sont légèrement supérieures aux valeurs théoriques qui sont de $1,3 \mathrm{mgCl}_{2} / \mathrm{L}$ (Légbassito) et $1,2 \mathrm{mgCl}_{2} / \mathrm{L}$ (Vakpossito).

Les Tableaux 8 et 9 donnent respectivement les valeurs théoriques attendues et obtenues ainsi que les résultats de désinfection des échantillons de teneurs maximales en nitrate de Légbassito et Vakpossito avec des taux d'abattement de $100 \%$ après le traitement. 


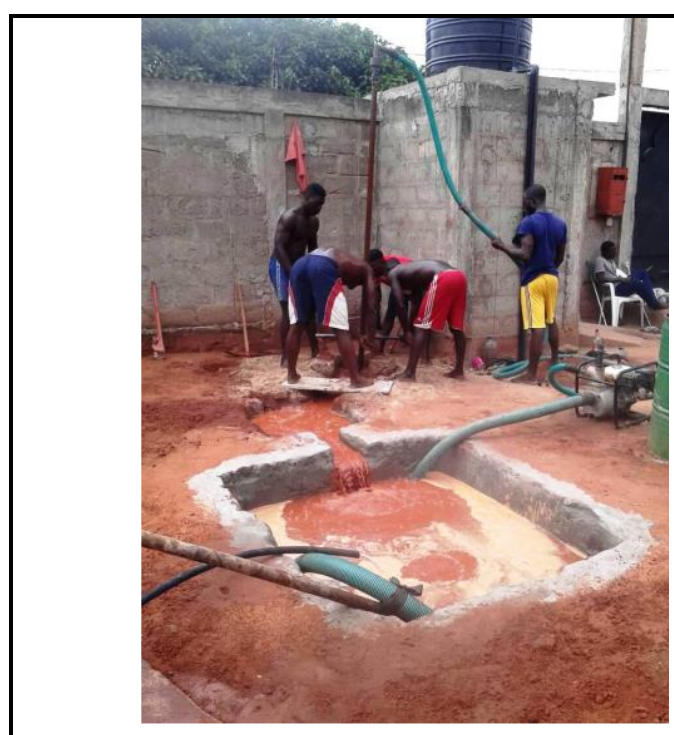

3a Forage manuel en cour de réalisation

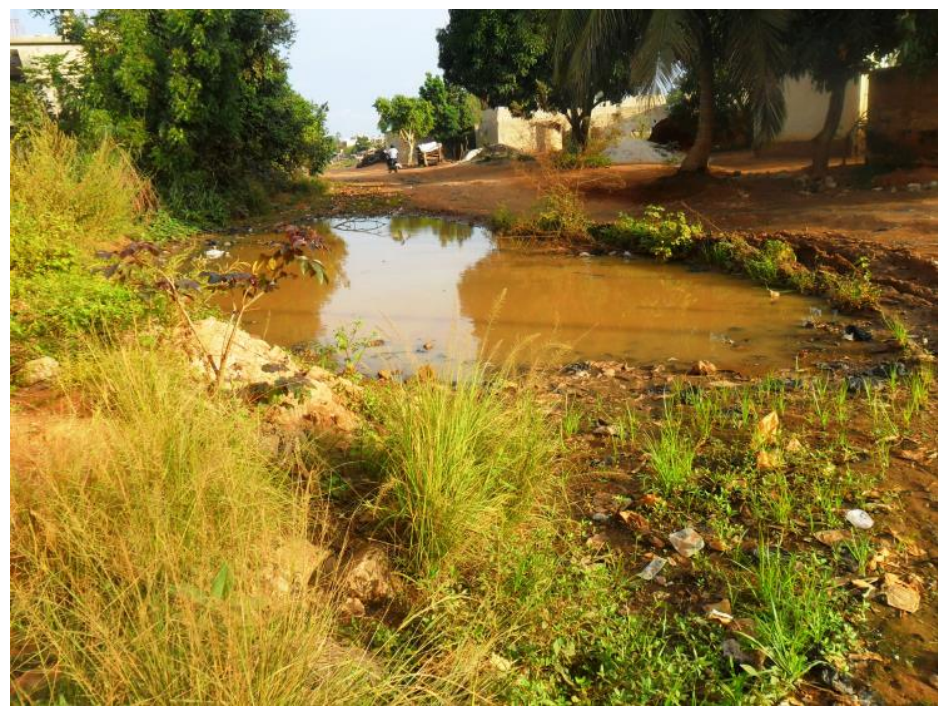

3c Eau stagnante sur une rue de Vakpossito

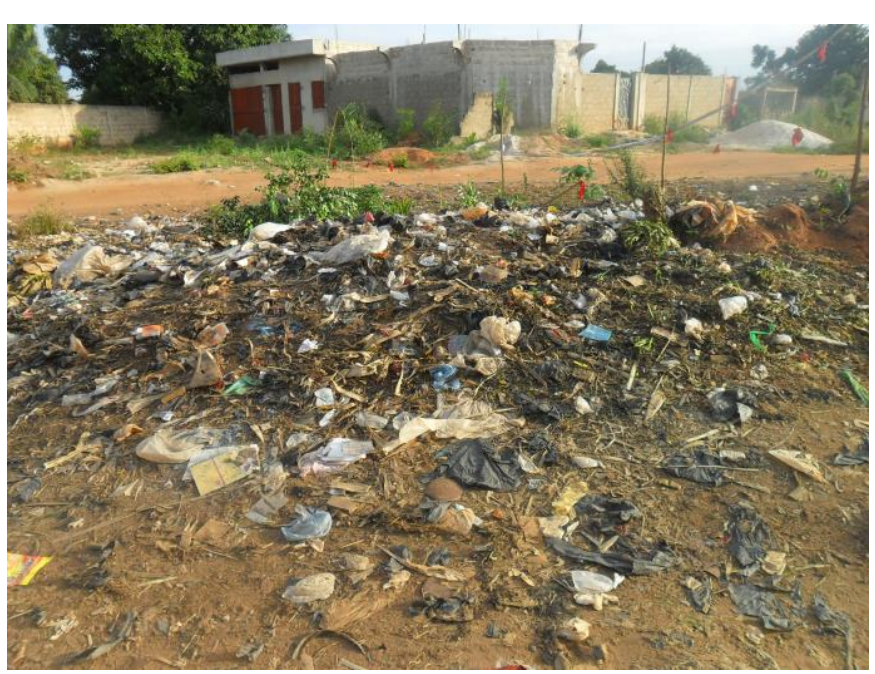

3b Dépotoir de quartier à Légbassito

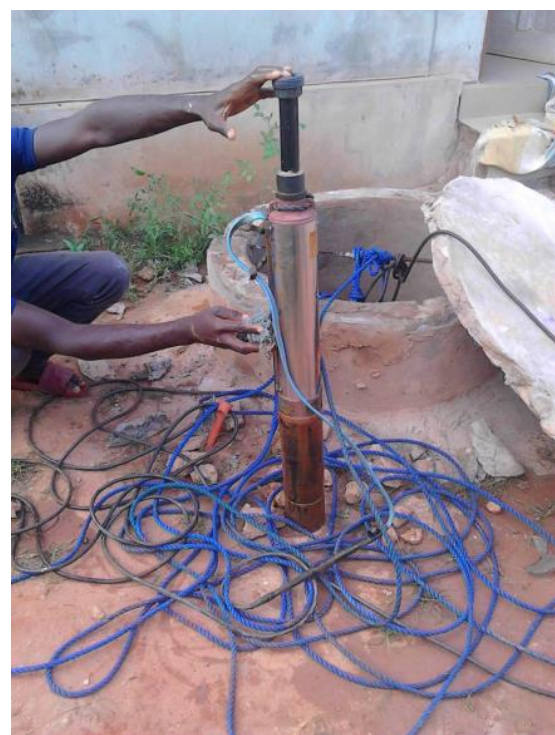

3d Entretient d'une pompe de forage

Figure 3 : Quelques aspects environnementaux et techniques des forages manuels.

Tableau 4 : Qualité physico-chimiques des eaux de forage.

\begin{tabular}{lcccc}
\hline Paramètres & Unité & Légbassito & Vakpossito & $\begin{array}{c}\text { Conc max. } \\
\text { OMS }(*)-\mathbf{U E}\end{array}$ \\
\hline Température & ${ }^{\circ} \mathrm{C}$ & $27-29$ & $26-29$ & 25 \\
$\mathrm{pH}$ & $\mathrm{upH}$ & $5,3-6,6$ & $4,9-6,1$ & $6,5-8,5$ \\
$\mathrm{CE}$ & $\mu \mathrm{S} / \mathrm{cm}$ & $305-1264$ & $471-1714$ & $400(\mathrm{NG})$ \\
$\mathrm{TDS}$ & $\mathrm{mg} / \mathrm{L}$ & $218-958$ & $337-1299$ & $1000(*)-1500$ \\
Turb & $\mathrm{NTU}$ & $0,3-8,8$ & $0,4-3,0$ & $<5$ \\
\hline
\end{tabular}




\begin{tabular}{lcccc}
\hline $\mathrm{HCO}_{3}{ }^{-}$ & $\mathrm{mg} / \mathrm{L}$ & $24,4-128,1$ & $12,2-73,2$ & $>30$ \\
$\mathrm{Cl}^{-}$ & $\mathrm{mg} / \mathrm{L}$ & $38,0-330,0$ & $95,1-480,5$ & 250 \\
$\mathrm{NO}_{3}{ }^{-}$ & $\mathrm{mg} / \mathrm{L}$ & $0,1-154,1$ & $12,1-121,6$ & $50\left(^{*}\right)-50$ \\
$\mathrm{SO}_{4}{ }^{2-}$ & $\mathrm{mg} / \mathrm{L}$ & $3,8-23,8$ & $4,0-26,6$ & $400(*)-250$ \\
$\mathrm{Na}^{+}$ & $\mathrm{mg} / \mathrm{L}$ & $21-142$ & $47-220$ & 150 \\
$\mathrm{~K}^{+}$ & $\mathrm{mg} / \mathrm{L}$ & $0,1-4,2$ & $0,7-4,8$ & 12 \\
$\mathrm{Ca}^{2+}$ & $\mathrm{mg} / \mathrm{L}$ & $11,2-68$ & $16,8-68$ & $100(\mathrm{NG})$ \\
$\mathrm{Mg}^{2+}$ & $\mathrm{mg} / \mathrm{L}$ & $2,9-38,4$ & $8,6-40,8$ & 50 \\
$\mathrm{Fe}^{2+}$ & $\mathrm{mg} / \mathrm{L}$ & $0,05-4$ & $\leq 0,05$ & $0,3(*)-0,2$ \\
$\mathrm{Mn}^{2+}$ & $\mathrm{mg} / \mathrm{L}$ & $\leq 0,05$ & $\leq 0,05$ & $0,4(*)-0,05$ \\
$\mathrm{IP}^{2+}$ & $\mathrm{mgO} / \mathrm{L}$ & $0,2-1,6$ & $0,2-0,9$ & $2(\mathrm{NG})$ \\
$\mathrm{SiO}_{2}$ & $\mathrm{mg} / \mathrm{L}$ & $16,5-170,4$ & $26,8-56,3$ & - \\
\hline
\end{tabular}

$\left(^{*}\right)$ : Directives de l'Organisation Mondiale de la Santé (OMS) ; NG : Nombre Guide ;CE :Conductivité électrique ;TDS : Solide Totaux dissous ; Tub :Turbidité.

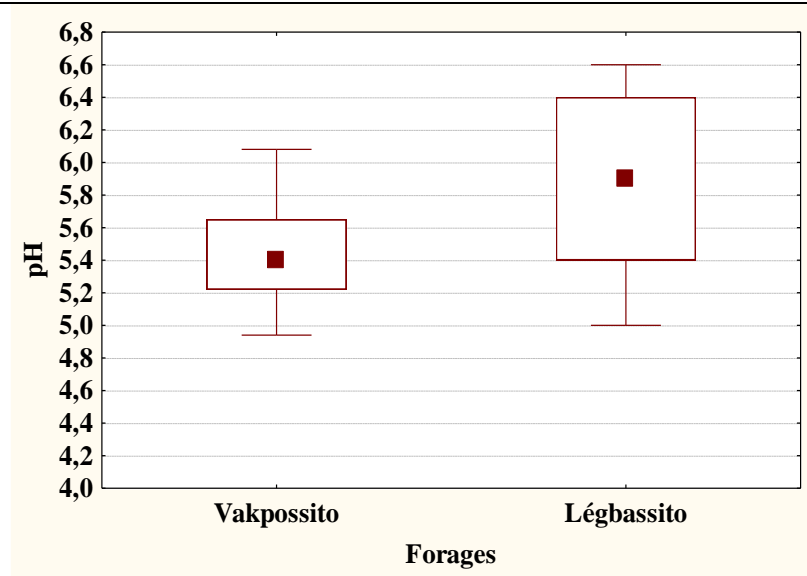

Médiane $\square$ 25\%-75\% I Etendue Non-Atypique ${ }^{\circ}$ Atypiques * Extrêmes $4 \mathrm{a}$

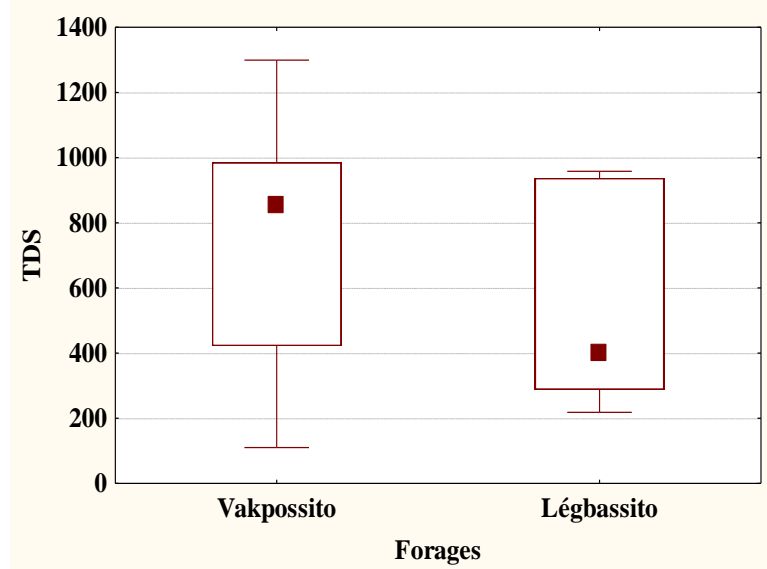

Médiane $\square$ 25\%-75\% I Etendue Non-Atypique ${ }^{\circ}$ Atypiques ${ }^{*}$ Extrêmes $4 \mathrm{c}$

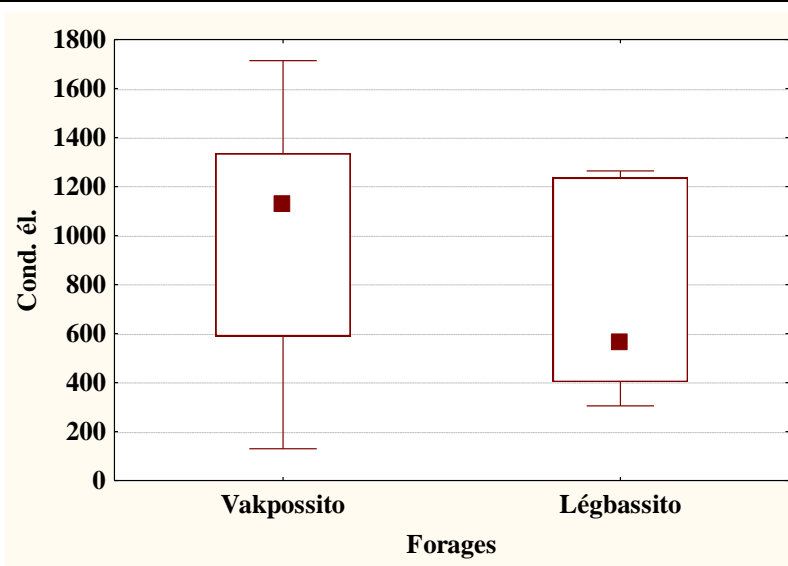

Médiane $\square$ 25\%-75\% I Etendue Non-Atypique ${ }^{\circ}$ Atypiques * Extrêmes $4 \mathrm{~b}$

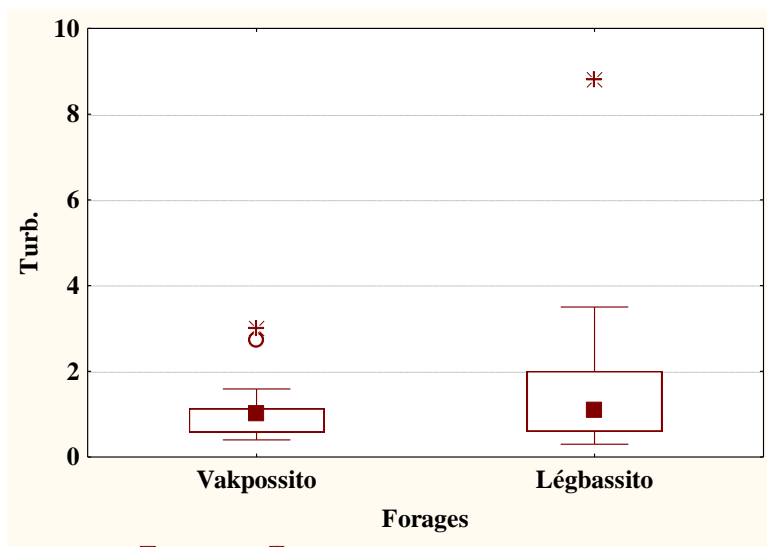

Médiane $\square$ 25\%-75\% I Etendue Non-Atypique ${ }^{\diamond}$ Atypiques $*$ Extrêmes $4 d$

Figure 4 : Variabilité spatiale du pH, de la conductivité électrique, et de la turbidité des eaux. 


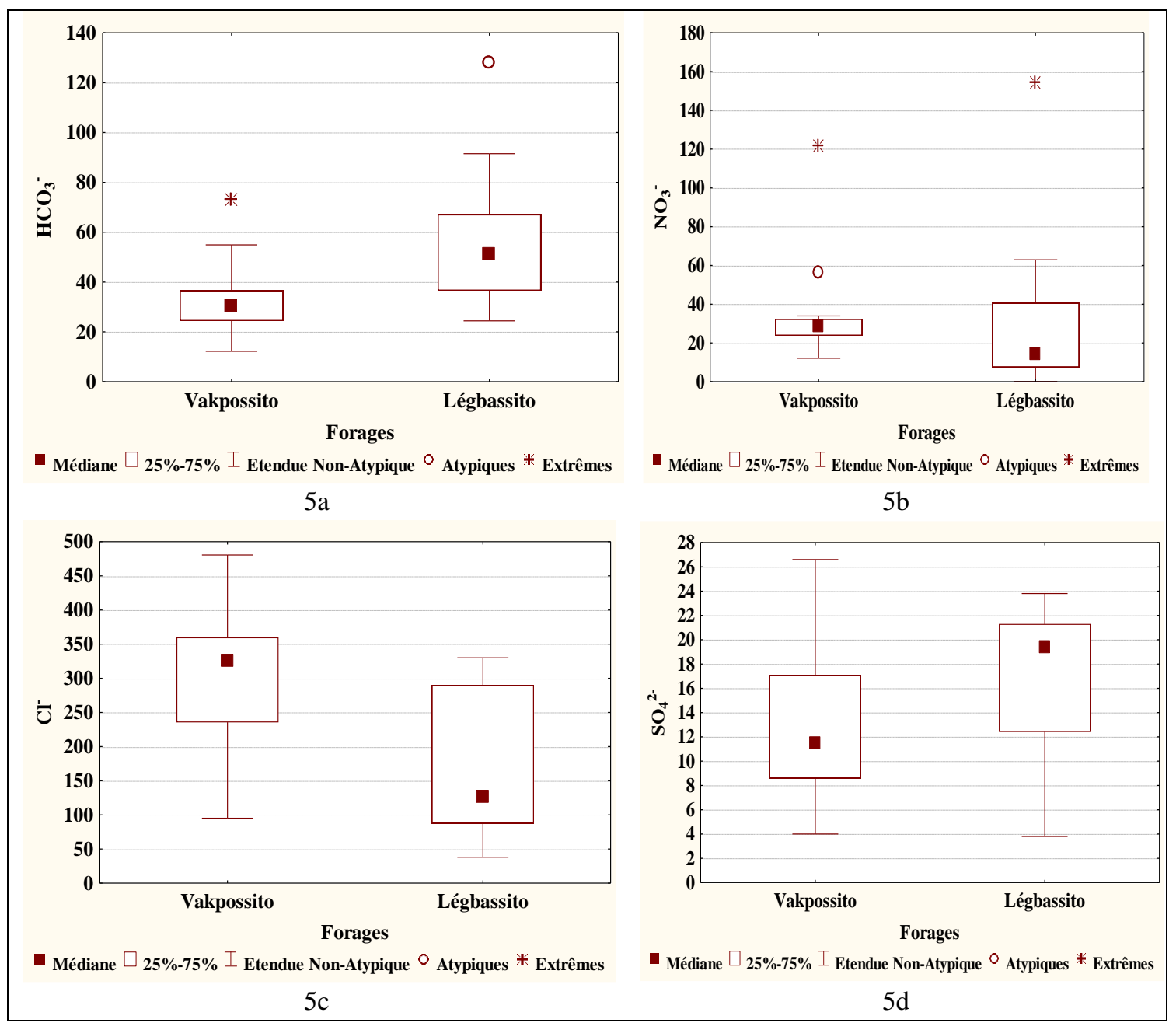

Figure 5 : Variabilité spatiale des ions $\mathrm{HCO}_{3}{ }^{-}, \mathrm{NO}_{3}{ }^{-}, \mathrm{Cl}^{-}$, et $\mathrm{SO}_{4}{ }^{2-}$.

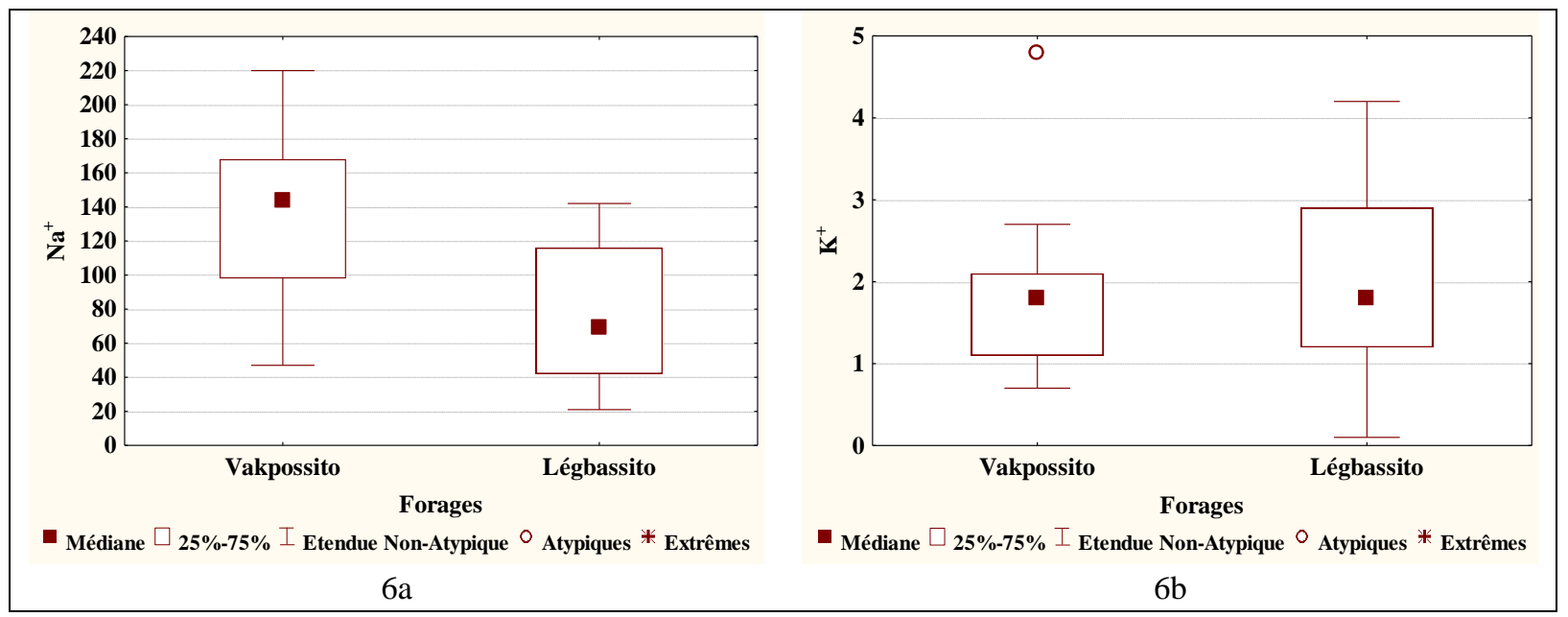




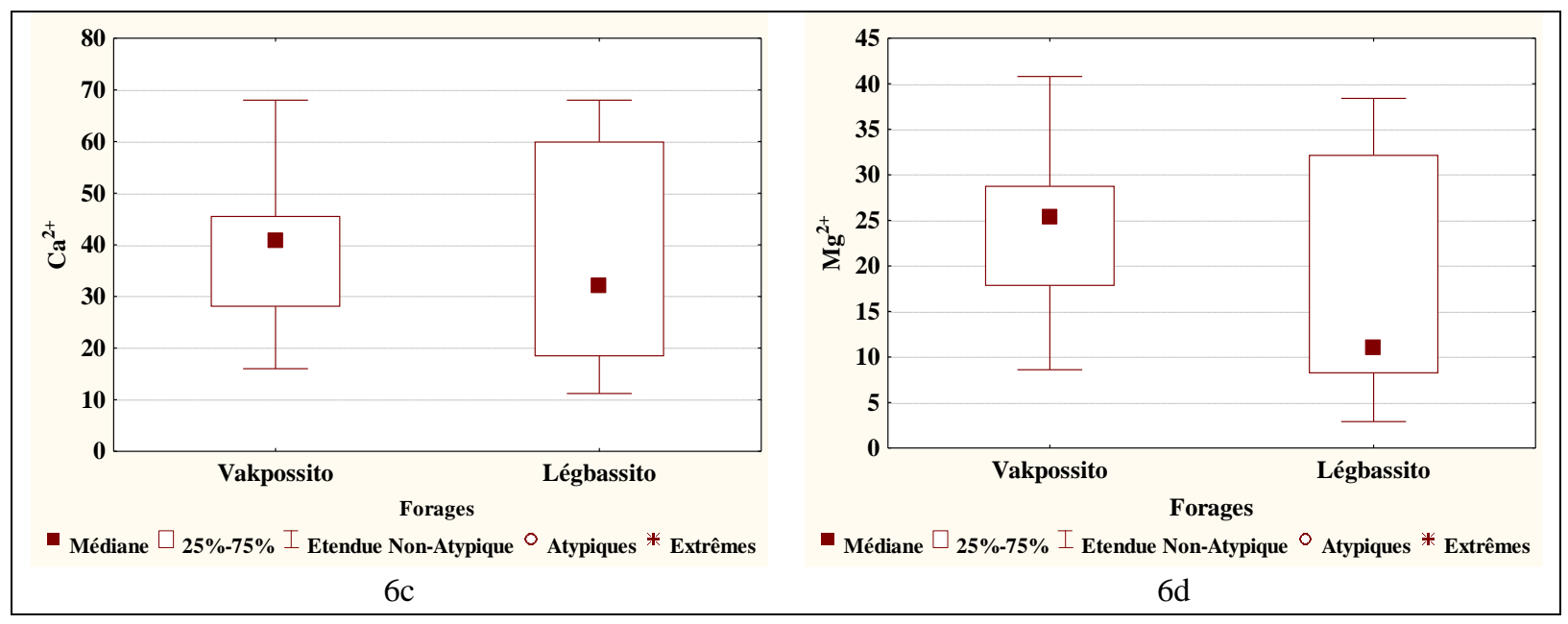

Figure 6 : Variabilité spatiale des ions $\mathrm{Na}^{+}, \mathrm{K}^{+}, \mathrm{Ca}^{2+}$ et $\mathrm{Mg}^{2+}$.

Tableau 5 : Résultats des analyses bactériologiques.

\begin{tabular}{lcccc}
\hline Paramètres & Unité & Légbassito & Vakpossito & Conc max. OMS (*) - UE \\
\hline Germes totaux & $\mathrm{ufc} / \mathrm{ml}$ & $98-2600$ & $68-4900$ & $10 \mathrm{ufc} / \mathrm{ml}$ \\
Coliformes totaux & $\mathrm{ufc} / \mathrm{ml}$ & $0-209$ & $0-709$ & $0 \mathrm{ufc} / 10 \mathrm{ml}$ \\
C. thermotolérants & $\mathrm{ufc} / \mathrm{ml}$ & $0-132$ & $0-115$ & $0 \mathrm{ufc} / 10 \mathrm{ml}$ \\
E. coli & $\mathrm{ufc} / \mathrm{ml}$ & $\mathrm{ND}$ & $\mathrm{ND}$ & $0 \mathrm{ufc} / 10 \mathrm{ml}$ \\
ASR & $\mathrm{ufc} / \mathrm{ml}$ & $0-8$ & $0-6$ & $0 \mathrm{ufc} / 20 \mathrm{ml}$ \\
\hline
\end{tabular}

ND : Non Déterminé ; C. Thermotolérants : Coliformes thermotolérants ; ASR : Anaérobies sulfitoréducteurs ; (*) Critères d'appréciation (AFNOR, 1997).

Tableau 6 : Matrice de corrélation.

\begin{tabular}{ccccccccccccccccc}
\hline Variables & $\mathrm{T}^{\circ} \mathrm{C}$ & $\mathrm{pH}$ & $\mathrm{C} n \mathrm{Ca}$ & $\mathrm{Ca}$ & $\mathrm{Mg}$ & $\mathrm{Na}$ & $\mathrm{K}$ & $\mathrm{HCO}$ & $\mathrm{Cl}$ & $\mathrm{SO} 4$ & $\mathrm{NO} 3$ & $\mathrm{GT}$ & $\mathrm{CTo}$ & $\mathrm{DFL}$ & $\mathrm{PrF}$ & $\mathrm{KMnO} 4$ \\
\hline $\mathrm{T} \mathrm{C}^{\circ}$ & $\mathbf{1 , 0 0}$ & $-0,16$ & $-0,23$ & $-0,28$ & $-0,19$ & $-0,25$ & $-0,27$ & $-0,21$ & $-0,19$ & $-0,29$ & $-0,14$ & $-0,02$ & $\mathbf{- 0 , 4 6}$ & $-0,19$ & $-0,03$ & $\mathbf{0 , 4 5}$ \\
$\mathrm{pH}$ & $-0,16$ & $\mathbf{1 , 0 0}$ & $-0,38$ & $-0,06$ & $-0,30$ & $-0,41$ & 0,17 & $\mathbf{0 , 7 6}$ & $-0,44$ & 0,36 & $-0,26$ & $-0,12$ & 0,19 & 0,30 & $-0,13$ & $-0,32$ \\
$\mathrm{Cond}$ & $-0,23$ & $-0,38$ & $\mathbf{1 , 0 0}$ & $\mathbf{0 , 7 9}$ & $\mathbf{0 , 9 0}$ & $\mathbf{0 , 9 4}$ & 0,29 & $-0,40$ & $\mathbf{0 , 9 7}$ & 0,09 & $\mathbf{0 , 5 4}$ & 0,06 & 0,17 & 0,21 & 0,11 & $-0,31$ \\
$\mathrm{Ca}$ & $-0,28$ & $-0,06$ & $\mathbf{0 , 7 9}$ & $\mathbf{1 , 0 0}$ & $\mathbf{0 , 9 2}$ & $\mathbf{0 , 6 5}$ & 0,43 & $-0,08$ & $\mathbf{0 , 7 0}$ & 0,21 & $\mathbf{0 , 5 5}$ & $-0,02$ & 0,06 & 0,43 & $-0,05$ & $-0,44$ \\
$\mathrm{Mg}$ & $-0,19$ & $-0,30$ & $\mathbf{0 , 9 0}$ & $\mathbf{0 , 9 2}$ & $\mathbf{1 , 0 0}$ & $\mathbf{0 , 7 8}$ & 0,33 & $-0,39$ & $\mathbf{0 , 8 4}$ & 0,11 & $\mathbf{0 , 6 0}$ & 0,06 & 0,01 & 0,39 & 0,00 & $-0,36$ \\
$\mathrm{Na}$ & $-0,25$ & $-0,41$ & $\mathbf{0 , 9 4}$ & $\mathbf{0 , 6 5}$ & $\mathbf{0 , 7 8}$ & $\mathbf{1 , 0 0}$ & 0,25 & $-0,42$ & $\mathbf{0 , 9 7}$ & 0,07 & 0,36 & 0,08 & 0,27 & 0,09 & 0,13 & $-0,29$ \\
$\mathrm{~K}$ & $-0,27$ & 0,17 & 0,29 & 0,43 & 0,33 & 0,25 & $\mathbf{1 , 0 0}$ & 0,27 & 0,24 & $\mathbf{0 , 5 0}$ & 0,20 & 0,00 & $-0,12$ & 0,08 & $-0,11$ & $-0,37$ \\
$\mathrm{HCO} 3$ & $-0,21$ & $\mathbf{0 , 7 6}$ & $-0,40$ & $-0,08$ & $-0,39$ & $-0,42$ & 0,27 & $\mathbf{1 , 0 0}$ & $\mathbf{- 0 , 4 8}$ & 0,13 & $-0,28$ & $-0,12$ & 0,21 & 0,09 & $-0,19$ & $-0,05$ \\
$\mathrm{Cl}$ & $-0,19$ & $-0,44$ & $\mathbf{0 , 9 7}$ & $\mathbf{0 , 7 0}$ & $\mathbf{0 , 8 4}$ & $\mathbf{0 , 9 7}$ & 0,24 & $\mathbf{- 0 , 4 8}$ & $\mathbf{1 , 0 0}$ & 0,07 & 0,38 & 0,10 & 0,14 & 0,12 & 0,14 & $-0,29$ \\
$\mathrm{SO} 4$ & $-0,29$ & 0,36 & 0,09 & 0,21 & 0,11 & 0,07 & $\mathbf{0 , 5 0}$ & 0,13 & 0,07 & $\mathbf{1 , 0 0}$ & 0,07 & $-0,33$ & 0,09 & 0,24 & $-0,09$ & $-0,43$ \\
$\mathrm{NO} 3$ & $-0,14$ & $-0,26$ & $\mathbf{0 , 5 4}$ & $\mathbf{0 , 5 5}$ & $\mathbf{0 , 6 0}$ & 0,36 & 0,20 & $-0,28$ & 0,38 & 0,07 & $\mathbf{1 , 0 0}$ & $-0,16$ & 0,18 & 0,33 & 0,09 & $-0,06$ \\
$\mathrm{GTo}$ & $-0,02$ & $-0,12$ & 0,06 & $-0,02$ & 0,06 & 0,08 & 0,00 & $-0,12$ & 0,10 & $-0,33$ & $-0,16$ & $\mathbf{1 , 0 0}$ & 0,00 & $-0,04$ & 0,32 & $-0,02$ \\
$\mathrm{CTo}$ & $\mathbf{- 0 , 4 6}$ & 0,19 & 0,17 & 0,06 & 0,01 & 0,27 & $-0,12$ & 0,21 & 0,14 & 0,09 & 0,18 & 0,00 & $\mathbf{1 , 0 0}$ & 0,05 & 0,16 & $-0,10$ \\
$\mathrm{DFL}$ & $-0,19$ & 0,30 & 0,21 & 0,43 & 0,39 & 0,09 & 0,08 & 0,09 & 0,12 & 0,24 & 0,33 & $-0,04$ & 0,05 & $\mathbf{1 , 0 0}$ & 0,05 & $-0,28$ \\
$\mathrm{PrF}$ & $-0,03$ & $-0,13$ & 0,11 & $-0,05$ & 0,00 & 0,13 & $-0,11$ & $-0,19$ & 0,14 & $-0,09$ & 0,09 & 0,32 & 0,16 & 0,05 & $\mathbf{1 , 0 0}$ & 0,11 \\
$\mathrm{KMnO} 4$ & $\mathbf{0 , 4 5}$ & $-0,32$ & $-0,31$ & $-0,44$ & $-0,36$ & $-0,29$ & $-0,37$ & $-0,05$ & $-0,29$ & $-0,43$ & $-0,06$ & $-0,02$ & $-0,10$ & $-0,28$ & 0,11 & $\mathbf{1 , 0 0}$ \\
\hline
\end{tabular}

Les valeurs en gras sont significativement différentes de 0 à un niveau de signification alpha $=0,05$. 


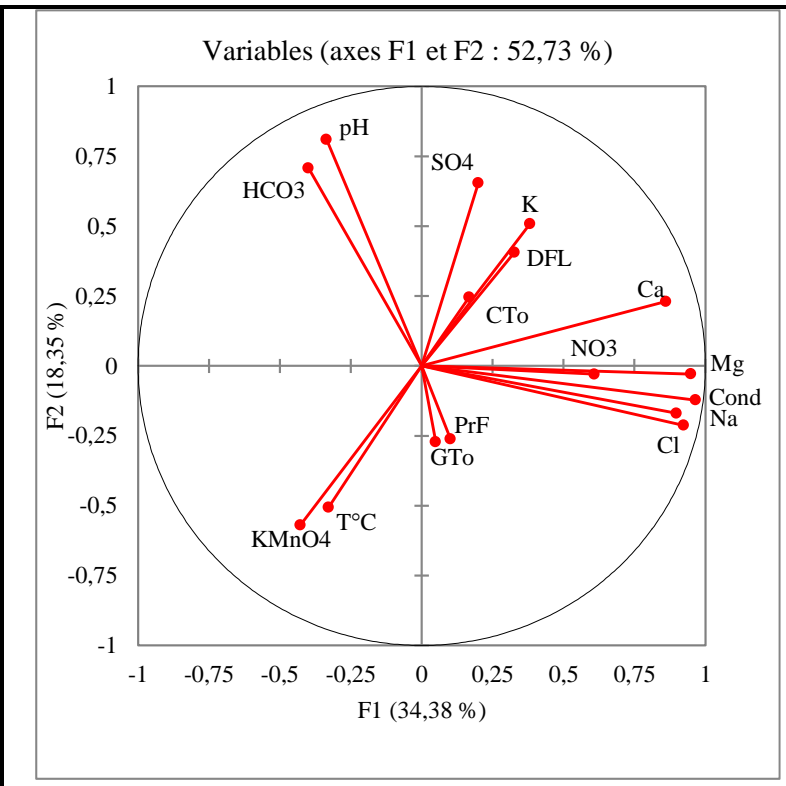

$7 \mathrm{a}$

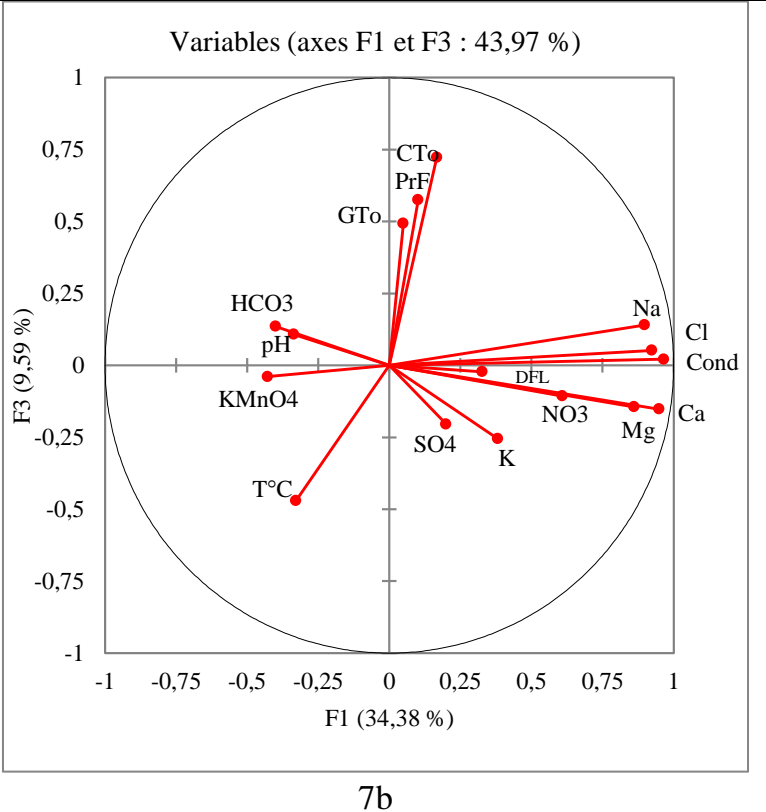

Figures 7 : Cercles de corrélation des paramètres.

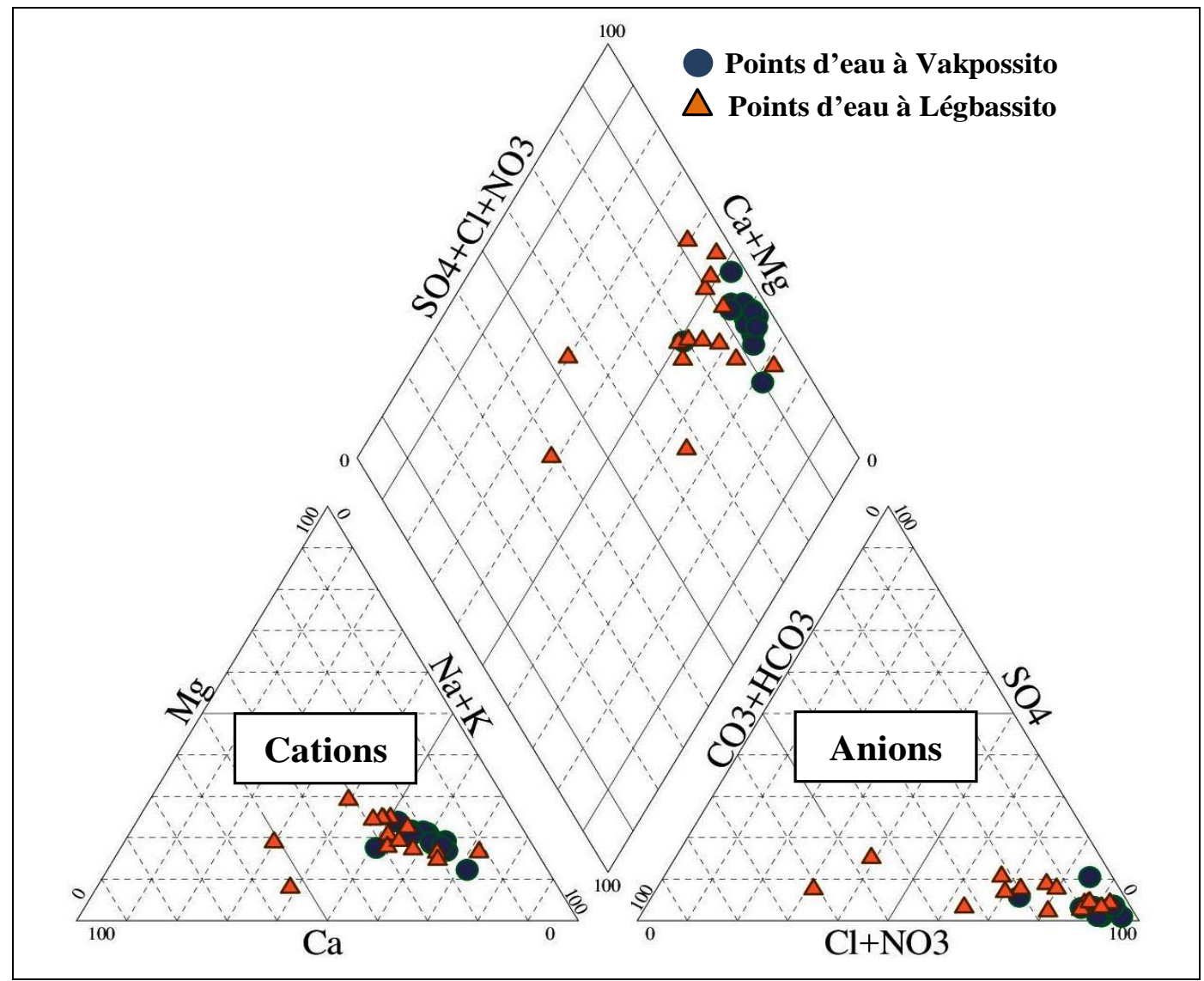

Figure 8 : Diagramme de Piper des eaux analysées. 

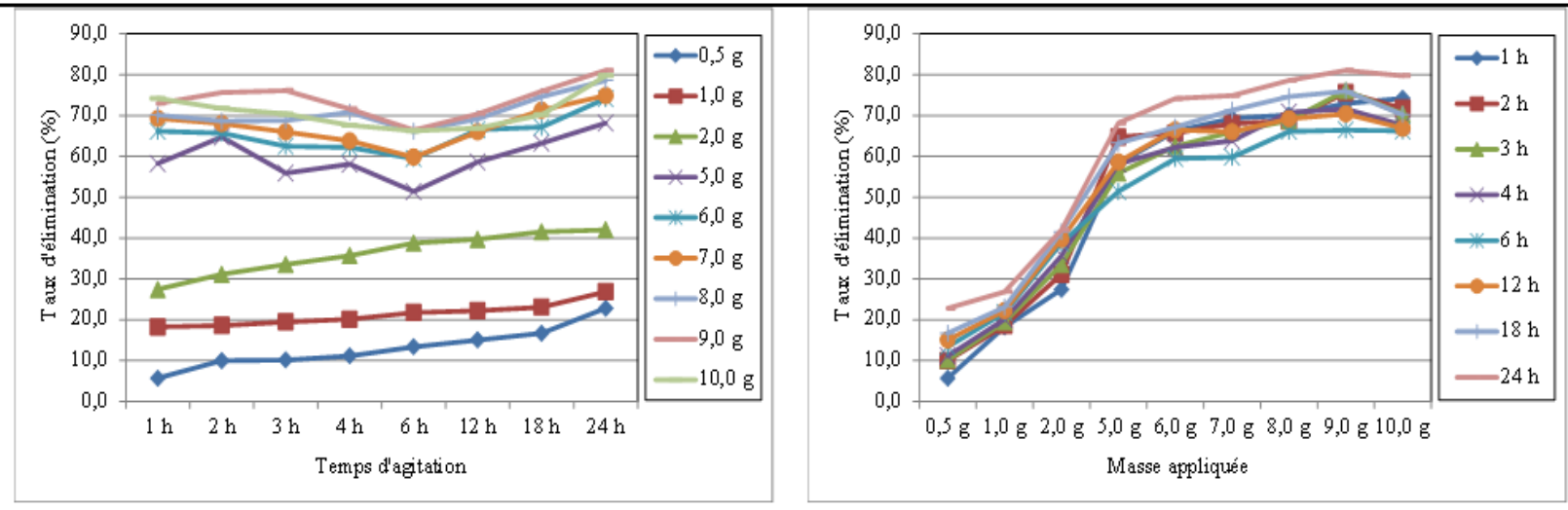

$9 \mathrm{a}$

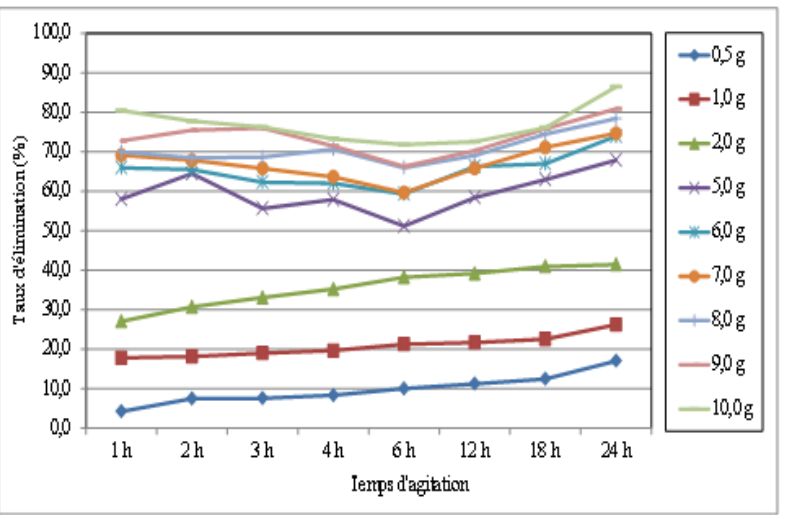

$9 \mathrm{c}$

$9 \mathrm{~b}$

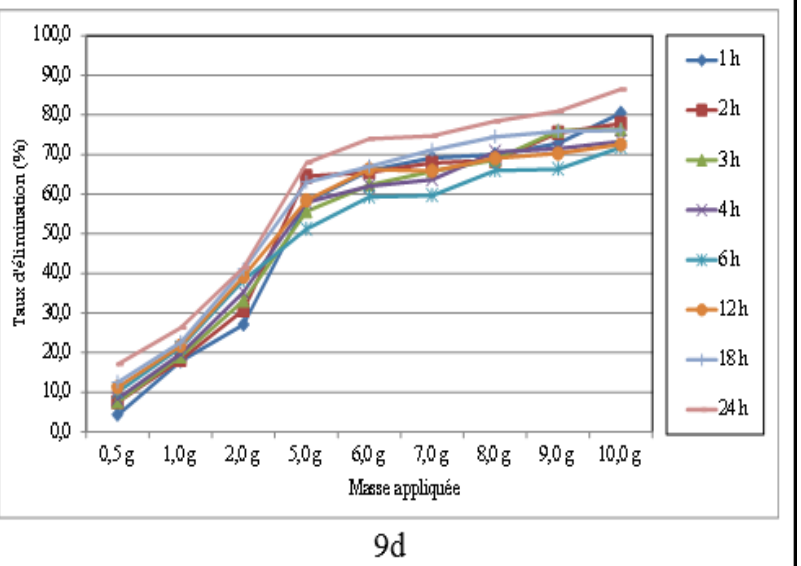

Figure 9: Taux d'élimination des nitrates par le CAP en fonction du temps et de la masse à Légbassito (Fig.9a et 9b) et à Vakpossito (Fig.9c et 9d).

Tableau 7 : Résultats d'élimination des nitrates.

\begin{tabular}{ccccc}
\hline Sites & Unités & Eau brute & Eau traitée & Taux d'abattement \\
\hline Légbassito & $\mathrm{mgNO}_{3}{ }^{-} / \mathrm{L}$ & 154,1 & 39,7 & $74,2 \%$ \\
Vakpossito & $\mathrm{mgNO}_{3}{ }^{-} / \mathrm{L}$ & 121,6 & 23,7 & $80,6 \%$ \\
\hline
\end{tabular}

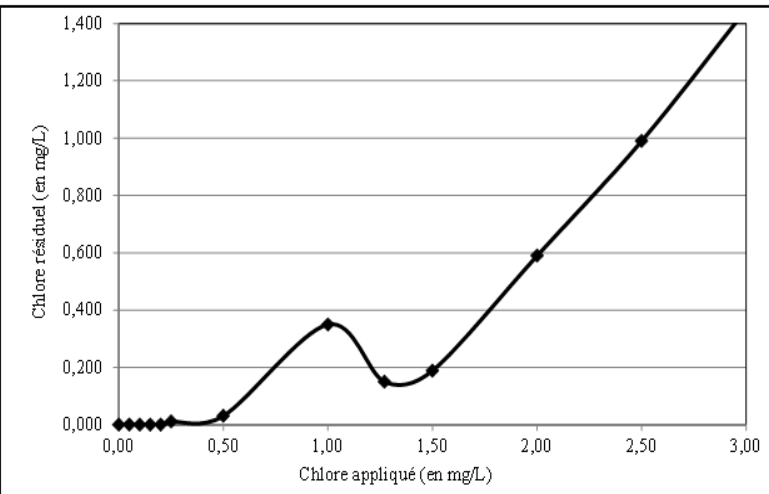

$10 \mathrm{a}$

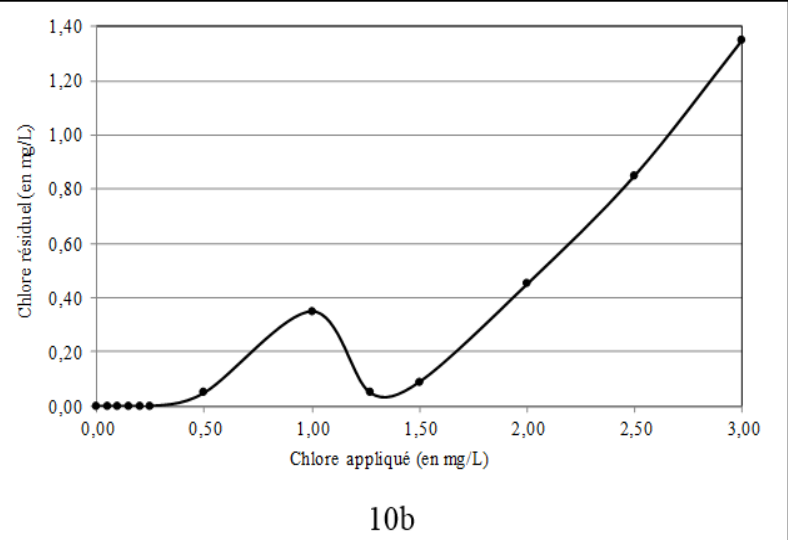

Figure 10 : Courbes de break point des échantillons de concentrations maximales en nitrate de Légbassito (10 a) et de Vakpossito (10 b). 
Tableau 8 : Valeurs théoriques attendues et obtenues de la demande en chlore.

\begin{tabular}{lcc}
\hline Echantillons & $\begin{array}{c}\text { Demande en chlore attendue } \\
(\mathbf{~ m g} / \mathbf{L})\end{array}$ & $\begin{array}{c}\text { Demande en chlore obtenue } \\
(\mathbf{m g} / \mathbf{L})\end{array}$ \\
\hline Légbassito & 1,30 & 1,35 \\
Vakpossito & 1,20 & 1,30 \\
\hline
\end{tabular}

Tableau 9: Résultats de désinfection des échantillons de teneurs maximales en nitrate de Légbassito et de Vakpossito.

\begin{tabular}{lllccc}
\hline Site & \multicolumn{1}{c}{ Paramètres considérés } & Unité & Eau brute & Eau traitée & Abattement \\
\hline \multirow{2}{*}{ Légbassito } & Germes totaux $\left(30^{\circ} \mathrm{C} / 72 \mathrm{~h}\right)^{(1)}$ & ufc $/ \mathrm{ml}$ & 766 & 0 & $100 \%$ \\
& Coliformes totaux $\left(30^{\circ} \mathrm{C} / 24 \mathrm{~h}\right)^{(2)}$ & ufc/ml & 209 & 0 & $100 \%$ \\
\hline \multirow{2}{*}{ Vakpossito } & Germes totaux $\left(30^{\circ} \mathrm{C} / 72 \mathrm{~h}\right)^{(3)}$ & $\mathrm{ufc} / \mathrm{ml}$ & 386 & 0 & $100 \%$ \\
& Coliformes totaux $\left(30^{\circ} \mathrm{C} / 24 \mathrm{~h}\right)^{(4)}$ & $\mathrm{ufc} / \mathrm{ml}$ & 15 & 0 & $100 \%$ \\
\hline
\end{tabular}

${ }^{(1)}$ Chlore appliquée : $1,35 \mathrm{mgCl}_{2} / \mathrm{L} ;{ }^{(2)}$ Chlore résiduel : $0,35 \mathrm{mgCl}_{2} / \mathrm{L}$.

${ }^{(3)}$ Chlore appliquée : $1,3 \mathrm{mgCl}_{2} / \mathrm{L} ;{ }^{(4)}$ Chlore résiduel : $0,44 \mathrm{mgCl}_{2} / \mathrm{L}$.

\section{DISCUSSION}

Accès à l'eau potable et les forages manuels

La problématique de l'accès à l'eau potable dans la ville de Lomé et ses banlieues a amené les populations à réaliser leur propre forage pour leur consommation et/ou vendu à des tiers. Cette pratique s'est installée au Togo depuis les années 2000. Selon les études dans le cadre du projet PRRESAF, on note en 2015 plus de 15000 forages recensés dans le Grand-Lomé (ville de Lomé et ses banlieues) dont 500 dans le canton Légbassito et Vakpossito (Rapport EAA-Togo, 2016 ; Fambi, 2018). La plupart des forages réalisés sont manuels et plus implantés dans le bassin sédimentaire côtier à cause des conditions géologiques et hydrogéologiques plus favorables (nappes phréatiques et aquifères du Continental Terminal, du Paléocène et du Maestrichtien plus accessibles) (Gnazou, 2008). Plus de $70 \%$ de forages manuels sont réalisés par les artisans exerçant dans le secteur informel et très peu de forages sont réalisés dans les règles de l'art (Rapport EAATogo, 2016 ; Fambi, 2018) (Figure 3a). Aussi, la qualité des eaux de ces forages manuels est rarement connue faute des analyses des eaux après leur réalisation.

\section{Qualité physicochimique des eaux}

Dans le cadre de cette étude, les eaux des forages choisis pour l'analyse (15 forages dans chaque canton Légbassito et Vakpossito) ont présenté pour la plupart des valeurs de $\mathrm{pH}$ à tendance acide selon les valeurs des médianes (Figure 4a). La médiane du pH de ces eaux est en dessous de 6,5. D'une façon générale on note une très faible variation de $\mathrm{pH}$ dans chaque zone et sur l'ensemble des points d'eau étudiés. Environ $10 \%$ des forages ont des valeurs de $\mathrm{pH}$ comprises entre 6,5 et 8,5 comme recommandé par l'OMS. En effet, la plupart des forages manuels sont réalisés dans les aquifères du Continental Terminal (profondeur 20 à $50 \mathrm{~m}$ ) qui est plus accessible pour les foreurs. La conductivité et la teneur en sels dissous expriment l'abondance des sels sous forment ionique et expriment l'état de minéralisation d'une eau (Rejesk, 2002 ; Coulibaly, 2005 ; OMS, 2011 ; UE, 2015). En effet, l'eau de la ville de Lomé et ses banlieues, surtout d'origine souterraine a une salinité comprise entre 700 et $1000 \mathrm{mg} / \mathrm{L}$ (TdE et ONEA, 2015 ; EAA-Togo, 2016). Les valeurs de la conductivité électrique et des sels dissous montrent une différence au sein d'un même site et d'un site à un autre ce qui est confirmé par le test de Kruskwal wallis (p 
$<0,05)$. On n'enregistre pas des valeurs extrêmes et atypiques au sein d'un même site (Figures $4 \mathrm{~b}$ et $4 \mathrm{c}$ ).

L'alcalinité d'une eau exprime principalement sa teneur en ions bicarbonates $\left(\mathrm{HCO}_{3}{ }^{-}\right)$, carbonates $\left(\mathrm{CO}_{3}{ }^{2-}\right)$ et hydroxyde $\left(\mathrm{OH}^{-}\right)$, et dépend entre autres, de la teneur en $\mathrm{CO}_{2}$, du $\mathrm{pH}$ et de la température de l'eau (Rodier, 2009 ; OMS, 2011). Les valeurs les plus élevées sont obtenues à Légbassito $(128,1$ $\mathrm{mg} / \mathrm{L}$ ) où la médiane se situe à $56,77 \mathrm{mg} / \mathrm{L}$. La distribution des valeurs au sein des sites montre qu'il y a une différence significative de l'alcalinité des eaux entre les sites, selon le test de variabilité de Kruskwal wallis ( $\mathrm{p}$ 0,05 ) (Figure 5a). Les variations des teneurs en nitrates observées au sein d'une même zone suggèrent l'influence de certains facteurs tels que l'hydrologie du milieu (ruissèlement, infiltration et zone de dépression) et les activités anthropiques (entretien périodique du forage et du réservoir, distance forage/latrinepuisard, présence de dépotoirs sauvages, etc.).

En général, outre leurs origines naturelles (marine, eaux de surface, dépôts atmosphériques, aérosols océaniques, eaux de pluie, percolations à travers les terrains gypsifères et salifères, etc.), les chlorures proviennent de nombreux rejets humains (urines, acide urique, etc.). L'ion chlorure possède des caractéristiques différentes de celles des autres éléments. Il n'est pas adsorbé par les formations géologiques, ne se combine pas facilement avec les éléments chimiques et reste très mobile (Coulibaly, 2005 ; Gnazou, 2008 ; Rodier, 2009 ; OMS, 2011). Les concentrations des ions sulfate dans les eaux naturelles sont généralement comprises entre 2 et $80 \mathrm{mg} / \mathrm{L}$, bien qu'elles puissent dépasser $1000 \mathrm{mg} / \mathrm{L}$ dans les rejets industriels ou dans les régions arides où des minéraux sulfatés tel que le gypse, sont présents. En considérant la position des médianes dans les deux sites, on constate néanmoins que la variation n'est pas négligeable, ce que confirme le test de Kruskwal wallis $(\mathrm{p}<0.05)$. Un écart de variation est observé dans le site de Légbassito $(3,8-23,8 \mathrm{mg} / \mathrm{L})$, et celui de Vakpossito $(4,0-26,6 \mathrm{mg} / \mathrm{L})$. Le titre hydrotimétrique exprime les teneurs en calcium $\left(\mathrm{Ca}^{2+}\right)$ et en magnésium $\left(\mathrm{Mg}^{2+}\right)$ d'une eau (Gnazou, 2008 ; Rodier, 2009). Ces résultats révèlent une contamination d'origine fécale de ces eaux. Ce qui indique un manquement aux règles d'hygiène.

L'observation des cercles montrent une corrélation entre les ions $\mathrm{Ca}^{2+}, \mathrm{Mg}^{2+}, \mathrm{Na}^{+}, \mathrm{Cl}^{-}$, $\mathrm{NO}_{3}{ }^{-}$et la conductivité électrique. Cette corrélation de la conductivité avec ces ions majeurs rend compte de la minéralisation ou du phénomène de l'hydrolyse des minéraux. Il faut noter que la conductivité décrit les sels inorganiques présents en solution dans l'eau (Ahoussi et al., 2010). Ces éléments peuvent provenir soit du milieu physique dans lequel l'eau a évolué, soit des rejets de certaines activités humaines dont l'eau est devenue le réceptacle (Kanohin et al., 2017); ceci contribue considérablement à la salinité des eaux. La forte corrélation entre les ions $\mathrm{Cl}^{-}$et $\mathrm{Na}^{+}\left(\mathrm{r}^{2}=0,97\right)$ serait à l'origine du goût salé de l'eau. Les résultats ont montré une corrélation entre le $\mathrm{pH}$ et $\left(\mathrm{HCO}_{3}{ }^{-}\right)\left(\mathrm{r}^{2}=0,76\right)$, ce qui montre que les ions $\mathrm{HCO}^{-}$contribuent à la régulation du $\mathrm{pH}$. Plus de $70 \%$ de ces eaux sont légèrement acide avec des valeurs de $\mathrm{pH}$ comprises entre 4,94 et 5,90. Cela est compatible avec le milieu silicaté qui constitue la nappe phréatique ou aquifère (Mbawala et al., 2010) La forte corrélation entre ions $\mathrm{Ca}^{2+}$ et $\mathrm{Mg}^{2+}, \quad\left(\mathrm{r}^{2}=0,92\right)$ proviendrait des minéraux silicatés des terrains et les formations traversées par les eaux de ruissellement et d'infiltration. Elle reflète également la dissolution des roches liée au temps de séjour de l'eau dans l'aquifère (Ahoussi al., 2010; Sadek, 2011) Les ions sulfate n'ont pas présenté de corrélation avec le calcium. Ils ne proviendraient pas de formations gypseuses, mais pourraient provenir probablement de l'oxydation de la pyrite (Gnazou, 2008). Il faut noter que la concentration en ions d'une eau ne reste pas toujours statique, elle est susceptible d'évolution en fonction du temps et de l'espace. On peut expliquer cette évolution par la nature des infiltrations qui ne sont pas identiques à tout moment, par les échanges 
ioniques entre les eaux et les formations argileuses lors de l'infiltration de l'eau à travers ces formations et d'autres facteurs (activités anthropiques autour du point d'eau).

En général, pour les mêmes conditions de l'expérience, on a noté que la concentration des nitrates augmente pour toutes les masses $(5 \mathrm{~g}$ à $10 \mathrm{~g})$, à partir de $3 \mathrm{~h}$ jusqu'à $12 \mathrm{~h}$ d'agitation. Cependant, les concentrations diminuent de 18 heures à $24 \mathrm{~h}$ et reste presque stable (Figures 9a, 9b, 9c et 9d). L'augmentation du taux des nitrates constatés entre 3 et 12 heures serait due à un phénomène de relargage (la remise en solution des nitrates préalablement fixés par le charbon actif). La lecture des Figures $9 b$ et $9 d$ montre qu'au fur et à mesure que la masse du charbon actif augmente, la concentration des nitrates diminuent. Les résultats des essais ont permis de déduire que le temps nécessaire est compris entre $1 \mathrm{~h}$ et $2 \mathrm{~h}$ ainsi que la masse de charbon pour une meilleure élimination des nitrates est de $100 \mathrm{~g} / \mathrm{L}$. Les concentrations de nitrate obtenues après le traitement sur les sites de Légbassito et de Vakpossito (respectivement $39,7 \mathrm{mg} / \mathrm{L}$ et $23,7 \mathrm{mg} / \mathrm{L}$ ) étaient toutes inférieures à la valeur guide de l'OMS (50 $\mathrm{mg} / \mathrm{L})$.

\section{Origine de la contamination}

L'appréciation de la qualité physicochimique et bactériologique des eaux de forages a été suivie par le biais de l'analyse de l'eau prélevée au niveau des 30 forages (15 forages à Légbassito et 15 à Vakpossito). La présence des coliformes thermotolérants dont Escherichia coli, ainsi que les Anaérobies sulfitoréducteurs ayant pour habitat naturel le tube digestif de l'homme et des animaux à sang chaux témoigne d'une contamination par les matières fécales (Prasad et Naranaya, 2004 ; de Guthe et Vanderweerd, 2006 ; Servais et al., 2007 ; Yapo et al., 2010 ; Soncy et al., 2015). Ainsi, la présence des germes de contamination d'origine fécale (Coliformes thermotolérants, et Anaérobies sulfitoréducteurs) en nombre élevé est présomptive de celle des pathogènes (Salmonelles et Vibrio cholerae). La contamination d'origine bactériologique des échantillons d'eau des forages pourrait avoir pour cause de la défécation dans la nature par les populations, la remonté du niveau piézométrique de la nappe phréatique, le lessivage des dépotoirs, l'écoulement et l'infiltration des eaux usées dans la nappe phréatique. Les germes retrouvés dans les eaux peuvent se développer dans le temps et affecter la qualité hygiénique et organoleptique de ces eaux. D'une façon générale, qu'une source de pollution soit proche du forage ou pas (puisard, fosse septique, dépotoir etc...), on a noté une contamination bactériologique d'origine fécale de la majorité de ces eaux (soit plus de $75 \%$ ) surtout pour les coliformes totaux témoignant $d$ 'un manque au règles d'hygiènes dans les ménages. Aussi, la contamination bactériologique serait due à une contamination ancienne des nappes lors de la réalisation des forages. De plus, l'entretien périodique de la pompe immergée (allant de 3 à 6 mois), pourrait être aussi une source de contamination périodique des eaux de forage.

Par ailleurs, la présence des composés minéraux azotés $\left(\mathrm{NO}_{3}{ }^{-}\right)$proviendrait aussi des mêmes sources de pollution, comme le lessivage des dépotoirs et des sols ainsi que l'écoulement et l'infiltration des eaux usées. La présence des nitrates en concentration élevées et la très faible teneur en ammonium et nitrite, pourraient s'expliquer par l'oxydation totale de l'azote ammoniacal. Cette oxydation passe par la formation des ammoniums, puis en nitrite et aboutit aux nitrates par le processus de nitrification (Rejsek, 2002 ; Rodier, 2009).

En général, la désinfection au chlore effectuée par les populations ne suit aucune étude préalable pour la détermination de la dose de chlore à appliquer. Ainsi, le traitement au chlore des eaux de forage dans la plupart des cas se révèle inefficace avec une contamination bactérienne fréquente et persistante à travers :

- Le renouvellement continu de l'eau de la nappe : formation géologique favorable à l'infiltration. 
- Une chloration irrégulière de l'eau de forage : dose de chlore non déterminée.

- Les forages non protégés : exposition aux vecteurs de contamination comme le vent, les eaux de ruissellement et les mouches.

- Le manque de suivi régulier de la qualité des eaux

\section{Conclusion}

Les eaux de forages constituent les sources d'alimentations en eau des populations des quartiers périphériques de Lomé. A cet effet l'évaluation de la qualité de ces eaux s'avère indispensable. Les forages manuels réalisés dans les zones péries urbaines (Vakpossito et Légbassito) sont proches des sources de pollution $(60 \%)$. Pour les paramètres $\mathrm{pH}$ acide, le taux des chlorures et goût salé respectivement de 90\% 56,66\% $86,67 \%$ des échantillons ne sont pas conformes à la norme OMS. Les concentrations des nitrates (élément toxique) et le fer (élément indésirable) sont respectivement très élevées dans $13 \%$ et $6 \%$ des eaux de forages analysées. Les analyses révèlent aussi la présence des coliformes fécaux dans 93,33\% des échantillons collectées. Cette pollution pourrait avoir pour cause: la non étanchéité des regards, la mauvaise implantation des puisards et latrines, le non-respect du périmètre de protection par rapport aux sources de pollution, le défaut de désinfection après la réalisation du forage et le faible respect des règles d'hygiène au cours des entretiens périodiques. Selon les résultats obtenus, les eaux souterraines des deux zones péries urbaines situées au Sud-Ouest du Togo sont impropres à la consommation. Au regard de ces résultats, ces eaux souterraines sont déclassées de l'utilisation comme eau de boisson. Force est de constater que les populations de ces zones utilisent ces eaux pour satisfaire au besoin hydrique. Les points d'eau ciblés sont des forages destinés à la vente publique d'eau. Une grande partie de la population s'expose à des risques en consommant ces eaux insalubres qui peuvent nuire à leur santé. Ceci représente un problème de santé publique que la population ignore. Pour réduire le degré de contamination de ces eaux, les essais de traitement réalisés ce sont révélés efficaces. Afin de prévenir les contaminations à partir des eaux de forages manuels des cantons de Vakpossito et de Légbassito, il convient de sensibiliser et de former les propriétaires de forage sur le traitement à base du charbon actif pour l'élimination des nitrates et la désinfection au chlore. Nous espérons que les résultats issus de ce travail permettront de sensibiliser les décideurs pour une meilleure application des normes de protection des forages en vue de la réduction de l'incidence des maladies liées à l'eau notamment les maladies diarrhéiques comme le choléra.

\section{CONFLITS D'INTERETS}

Les auteurs déclarent qu'ils n'ont pas d'intérêts concurrents.

\section{CONTRIBUTIONS DES AUTEURS}

Les campagnes d'échantillonnage et les analyses ont vu la participation de GB, MA et KF. La rédaction du manuscrit a vu la contribution de GD, LMB, SKS, MA et KF.

\section{REFERENCES}

2iE. 2011. Manuel technique de la gestion intégrée des ressources en eau en Afrique subsaharienne.142p.

AFNOR. 1997. Eaux : méthode d'Essai. Directive relative à la qualité des eaux destinées à la consommation humaine ; directive 80/777/ CEE du 15juillet 1980. Ed Afnor Paris. 624p.

Ahoussi KE, Soro N, Koffi YB, Soro G, Biemi J. 2010. Origine de la minéralisation des eaux des aquifères discontinus sous couvert forestier de la zone Sud de la Côte d'Ivoire : cas de la région d'Abidjan-Agboville. Int. J. Biol. Chem. Sci., 4(3): 782-797. http://ajol.info/index.php/ijbcs

Ajibade WA, Ayodele IA, Agbede SA. 2008. Microbiological characteristics of waters in the major rivers in Kanji Lake. African journal of Environmental Science and Technology, 2(8): 208-216. 
DOI:

https://doi.org/10.5897/AJEST.9000075

Akouvi A, Dray M, Violette S, de Marsily GH, Zuppi GM. 2008. The sedimentary coastal basin of Togo : example of a multilayered aquifer still influenced by a palaeo-seawater intrusion. Hydrogeology Journal, 16: 419-436. DOI : 10.1007/s10040-007-0246-1

ARC Ingénieur. 2016. Projet de Renforcement de l'Alimentation en eau potable en milieu Urbain : Etude d'Avant-Projet Détaillé (APD), volume 1-mémoire descriptif technique du projet, 109 pages.

Ayah M. 2016. Contribution des apports anthropiques dans la pollution d'un hydrosystème lagunaire subtropical : hydrogéochimie du système lagunaire de Lomé (Togo). Thèse de doctorat. FDS.UL. 204p + annexes.

Balogoun KC. 2016. Valorisation en charbon actif par voie chimique des coques de Coco nucifera et des sons d'Oriza sativa : caractérisation et application en traitement des eaux. Thèse de doctorat à l'université d'Abomey- Calavi (Bénin). $213 p+$ annexes

Bhatnagar A, Sillanpää FE. 2011. A review of emerging adsorbents for nitrate removal from water. Chemical Engineering Journal, 168: 493-504. DOI: https://doi.org/10.1016/j.cej.2011.01.103

Chen Y, Zhu Y, Wang Z, Li Y, Wang L, Ding L, Gao X, Ma Y, Guo Y. 2011. Application studies of activated carbon derived from rice husks produced by chemical-thermal process - A review. Advances in Colloid and Interface Science, 163: 39-52. DOI: https://doi.org/10.1016/j.cis.2011.01.006

Coulibaly K. 2005. Etude de la qualité physico-chimique et bactériologique de l'eau des puits de certains quartiers du district de Bamako. Thèse de doctorat en médecine Université de Bamako (Mali), Faculté de Médecine de Pharmacie et d'Odontostomatologie ; 269p.

da Costa YP. 2005. Biostratigraphie et Paléogéographie du bassin sédimentaire du Togo. Thèse Doct., Univ. de Lomé, 2 tomes, 476p.

de Guthe CV, Vanderweerd V. 2006. Sommet Mondial sur le Développement Durable : Objectif assainissement. 8p.

DGSCN. 2011. Rapport sur les résultats du 4ème Recensement Général de la Population et de l'Habitat (4ème RGPH, 2010). Bureau central du recensement (Togo), 65p.

EAA-Togo-UE. 2016. Projet de Réduction de Risques Environnementaux et Sanitaires liés à l'Activité des vendeurs d'eau de Forage de la ville de Lomé (PRRESAF).

Fahmy T. 2006. Addinsoft, 1993-206. www.xlstat.com

Fambi K. 2018. Contribution à la potabilisation des eaux de forages dans les zones périurbaines : cas des cantons de Légbassito et de Vakpossito, Lomé (Togo). Mémoire de Master Institut International d'Ingénierie de l'Eau et de l'Environnement (2iE), Ouagadougou, Burkina Faso, 71p.

Gnazou MDT. 2008. Etude hydrodynamique, hydrogéochimique, isotopique et modélisation de l'aquifère du paléocène du bassin sédimentaire côtier du Togo. Thèse de doctorat. FDS.UL. 204p+annexes.

Groupement SGI Ingénieurs SA - Hydro R\&D - SOTED Afrique. 2003. Etudes d'assainissement de la ville de Lomé et d'alimentation en eau potable et assainissement de 20 centres semi urbains. Etudes institutionnelles ; phase A : collecte des données de base. Volume 1 : Rapport. Edition définitive. $285 p$.

GSE (Groupe Scientifique sur l'Eau). 2003. Nitrates/Nitrites. Fiches synthèses sur l'eau potable et la santé humaine. Institut National de Santé Publique du Québec, $12 \mathrm{p}$.

Kanohin F, Yapo OB, Dibi B, Bonny AC. 2017. Caractérisation physico-chimique et bactériologique des eaux souterraines de Bingerville. Int. J. Biol. Chem. Sci., 
11(5): 2495-2509. DOI: https://dx.doi.org/10.4314/ijbcs.v11i5.43

Mbawala A, Abdou, Ngassoum MB. 2010. Evaluation de la pollution physicochimique et microbienne des eaux de puits de Dang-Ngaoundéré (Cameroun). Int. J. Biol. Chem. Sci., 4(6): 1962-1975. http://ajol.info/index.php/ijbcs

OMS. 2011. Directives de Qualité pour l'Eau

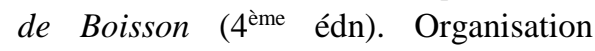
Mondiale de la Santé: Genève, Suisse ; 307-447.

Prasad BG, Narayana TS. 2004. Subsurface water quality of different sampling stations with some selected parameters at Machilipatnam Town. Nat. Env. Poll. Tech., 3(1) : 47-50.

Rejsek F. 2002. Analyse des eaux. Aspects réglementaires et techniques, Série Sciences et techniques de l'environnement, Centre régional de documentation pédagogique d'Aquitaine, Bordeaux, France. 368p.

Rodier J. 2009. L'Analyse de l'Eau: Eaux Naturelles; Eaux Résiduaires, Eaux de

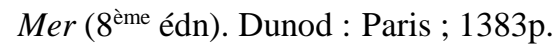

Sadek A. 2011. Hydrochimie et faciès géochimiques des eaux souterraines, Plaine de Bekaa. Hydrological Sciences Journal, 56(2): 334-348. DOI: 10.1080/02626667.2011.559331.

Segniagbeto H. 2009. Herpetofaune du Togo : taxonomie, Biogéographie. Thèse de Doct. Univ. Lomé. Tommes I et II. 363 p.

Servais P, Garcia-Armisen T, George I, Billen G. 2007. Fecal bacteria in the rivers of the Seine drainage network (France): sources, fate and modeling. Sciences of the Total Environment: 16p. DOI: https://doi.org/10.1016/j.scitotenv.2006. 12.010

Simler R. 2005. Logiciel d'hydrochimie. http// :www.lha.univavignon.fr/logiciel.h tm
Soncy K, Djeri B, Anani K, Eklou-Lawson M, Adjrah Y, Karou DS, Ameyapoh Y, de Souza C. 2015. Evaluation de la qualité bactériologique des eaux de puits et de forage à lomé, togo. Journal of Applied Biosciences, 91: 8464-8469. DOI: 10.4314/jab.v91i1.6

Tchakala I. 2008. Contribution à l'étude de la demande en chlore des eaux dans la zone à forte densité. Mémoire de DEA FDS/UL ; 60p.

Tchakala I. 2013. Contribution à l'étude de la préparation des charbons actifs à partir des résidus carbonés des industries de transformation agroalimentaire (cas des tourteaux de karité et des tourteaux de de coton) : caractérisation et applications en traitement des eaux. Thèse de Doctorat, université de Lomé, Togo, 198p.

TdE-ONEA. 2015. Rapport Plan d'Amélioration des Performances (PAP) de Moyen Terme période de 2015 à 2018, Société Togolaise des Eaux (TdETogo) et l'Office National de l'Eau et de l'Assainissement (ONEA-Burkina-Faso), 192p.

UE. 2015. Directive (UE) $\mathrm{n}^{\circ}$ 2015/1787 du 06/10/15 modifiant les annexes II et III de la directive98/83/CE du Conseil relative à la qualité des eaux destinées à la consommation humaine.

Yapo OB, Mambo V, Seka A, Ohou MJA, Konan F, Gouzile V, Tidou AS, Kouame KV, Houenou P. 2010. Évaluation de la qualité des eaux de puits à usage domestique dans les quartiers défavorisés de quatre communes d'Abidjan (Côte d'Ivoire) : Koumassi, Marcory, Port-Bouet et Treichville. Int. J. Biol. Chem. Sci., 4(2) : 289-307. DOI: 10.4314/ijbcs.v4i2.58111. 